NBER WORKING PAPER SERIES

\title{
THE PRICE IMPACT AND SURVIVAL OF IRRATIONAL TRADERS
}

\author{
Leonid Kogan \\ Stephen Ross \\ Jiang Wang \\ Mark Westerfield \\ Working Paper 9434 \\ http://www.nber.org/papers/w9434
}

\author{
NATIONAL BUREAU OF ECONOMIC RESEARCH \\ 1050 Massachusetts Avenue \\ Cambridge, MA 02138 \\ January 2003
}

The authors thank Ming Huang and participants of the 2002 NBER Asset Pricing Summer Conference and seminars at LBS, University of Minnesota, NYU, and MIT for valuable comments. The views expressed herein are those of the authors and not necessarily those of the National Bureau of Economic Research.

(C) 2003 by Leonid Kogan, Stephen Ross, Jiang Wang, and Mark Westerfield. All rights reserved. Short sections of text not to exceed two paragraphs, may be quoted without explicit permission provided that full credit including, (C) notice, is given to the source. 
The Price Impact and Survival of Irrational Traders

Leonid Kogan, Stephen Ross, Jiang Wang, and Mark Westerfield

NBER Working Paper No. 9434

January 2003

JEL No. G1, G0

\section{$\underline{\text { ABSTRACT }}$}

Milton Friedman argued that irrational traders will consistently lose money, won't survive and, therefore, cannot influence long run equilibrium asset prices. Since his work, survival and price influence have been assumed to be the same. Often partial equilibrium analysis has been relied upon to examine the survival of irrational traders and to make inferences on their influence on prices. In this paper, we demonstrate that survival and influence on prices are two independent concepts. The price impact of irrational traders does not rely on their long-run survival and they can have a significant impact on asset prices even when their wealth becomes negligible. In addition, in contrast to a partial equilibrium analysis, general equilibrium considerations matter since the ability of irrational traders to impact prices even when their wealth is diminishing can significantly affect their chances for long-run survival. In sum, in a long-run equilibrium, we explicitly show that price impact can occur whether or not the irrational traders survive. In related work, we show that even if the irrational traders survive they may have no price impact.

Leonid Kogan

Sloan School of Management

MIT, E52-434

50 Memorial Drive

Cambridge, MA 02142

and NBER

lkogan@mit.edu

Jiang Wang

Sloan School of Management

MIT, E52-456

50 Memorial Drive

Cambridge, MA 02142

and NBER

jwang@mit.edu
Stephen Ross

Sloan School of Management

MIT, E52-443

50 Memorial Drive

Cambridge, MA 02142

and NBER

sross@mit.edu

Mark Westerfield

Department of Economics

MIT, E52

50 Memorial Drive

Cambridge, MA 02142

mmwester@mit.edu 


\section{Contents}

1 Introduction $\quad 1$

2 The Model $\quad 3$

$\begin{array}{llr}3 & \text { The Equilibrium } & 6\end{array}$

4 A Special Case: Logarithmic Preferences $\quad 8$

5 The Survival of Irrational Traders $\quad 14$

5.1 Heuristic Partial Equilibrium Analysis . . . . . . . . . . . . . . 15

5.2 General Equilibrium Analysis . . . . . . . . . . . . . . . 16

5.3 Absolute Extinction . . . . . . . . . . . . . . . . . 20

6 The Price Impact of Irrational Traders 20

7 Portfolio Policies $\quad 23$

8 Conclusion $\quad 28$

A Wealth Distribution and Welfare $\quad 30$

$\begin{array}{ll}\text { B Proofs } & 33\end{array}$ 


\section{Introduction}

Most neoclassical asset pricing models rely on the assumption that market participants (traders) are rational in the sense that they behave in ways that are consistent with the objective probabilities of the states of the economy (e.g., Radner (1971) and Lucas (1978)). More particularly, they maximize expected utilities using the true probabilities of uncertain economic states. This approach is firmly rooted in the tradition of going from the normative to the positive in economics, yet there is mounting evidence that it is not descriptive of the observed behavior of the average market participant (see, e.g., Alpert and Raiffa (1959), Benartzi and Thaler (2001), Black (1986), Kahneman and Tversky (1979), Odean 1998)). How the presence of traders with incorrect beliefs may affect the behavior of financial markets remains an open question.

It has long been argued (see, e.g., Friedman (1953)) that irrational traders who use wrong beliefs cannot survive in a competitive market. Trading under the wrong beliefs makes them lose money to the rational traders and eventually lose their wealth. It is the rational traders who in the long-run control most of the wealth and determine asset prices. Using a partial equilibrium model, De Long, Shleifer, Summers and Waldmann (1991) suggest that traders with wrong beliefs may survive in the long run because they may hold portfolios with higher growth rates and therefore can eventually outgrow the rational traders. ${ }^{6}$ In contrast, in a general equilibrium setting, Sandroni (2000) and Blume and Easley (2001) show that with intermediate consumption, irrational traders do not survive in the long-run.

The efficiency of financial markets is the principal motivation behind the interest in the survival of irrational traders. If irrational traders impact asset prices, then markets will not be efficient, either informationally or allocationally. Implicitly, the discussion on survival is based on the assumption that survival is a necessary condition for long-run price impact. It is thought that irrational traders have to control a significant amount of wealth in order to affect - or 'infect' - prices with their irrational beliefs. In this paper, we show that this assumption is false and that irrational traders can maintain a large price impact even as their relative wealth diminishes towards zero over time.

Our analysis is conducted with a parsimonious general equilibrium model inhabited by

\footnotetext{
${ }^{6}$ See also Figlewski (1978) for a discussion on the notion of long-run survival. Many papers in existing literature analyze survival of irrational traders and their effect on prices in financial markets with various constraints and imperfections, e.g., De Long, Shleifer, Summers and Waldmann (1990), Shleifer and Vishny (1997), Hirshleifer, Subrahmanyam, Titman (2002). In contrast, the setting of this paper assumes perfect, complete financial markets.
} 
both rational traders and irrational traders. Traders only care about their terminal consumption. We are able to derive an explicit solution to the model, and we obtain conditions under which the irrational traders can survive in the long run in the sense that their share of the total wealth does not go to zero over time. However, we show that even when irrational traders do not survive, with a negligible amount of wealth they can still exert significant influence on the asset price over a long period of time.

Underlying this initially counterintuitive result is a solid economic intuition. Under incorrect beliefs, irrational traders express their views by taking positions (bets) on extremely unlikely states of the economy. As a result, the state prices of these extreme states can be significantly affected by the beliefs of the irrational traders with negligible wealth. In turn, these states, even though highly unlikely, can have a large contribution to current asset prices. This is especially true for states associated with extremely low levels of aggregate consumption in which the traders' marginal utilities are very high and so too are the state prices. The beliefs of the irrational traders on these low probability but high marginal utility states can influence current asset prices and their dynamics. Furthermore, irrational traders need not take extreme positions in order to influence prices. The formal analysis clearly verifies this conceptual distinction between the long-run price impact and the longrun survival of irrational traders.

The possibility that irrational traders may have a significant price impact with a negligible share of wealth also has important implications for their survival. In the partial equilibrium analysis of De Long, Shleifer, Summers and Waldmann (1991) (DSSW, thereafter), for example, it was assumed that when the irrational traders control only a negligible fraction of the total wealth, they have no impact on asset prices, i.e., asset prices behave as if the irrational traders are absent. Given the rationally determined prices, DSSW then show that the wealth of irrational traders can grow at a faster rate than the wealth of the rational traders. Thus the irrational traders can recover from their losses and survive in the longrun. Although such an argument is illuminating, the limitations of partial equilibrium do not allow it to adequately resolve the question of price impact. As we have argued, irrational traders may still influence prices with diminishing wealth. Moreover, such a possibility can significantly affect the irrational traders' portfolio policies in ways that make their recovery difficult.

The paper proceeds as follows. Section 2 develops a canonical, pure exchange economy similar to that of Black and Scholes (1973), but in presence of irrational traders who have persistently wrong beliefs about the economy, and Section 3 describes the general equilibrium 
of this economy. Section 4 treats the special case of logarithmic preferences and demonstrates that although irrational traders never survive they nevertheless can still influence long-run asset prices. Section 5 analyzes the survival of irrational traders for the general case. We first consider the partial equilibrium arguments of DSSW using our setting to analyze the conditions under which the irrational traders may survive in the long run. We then present the general equilibrium results on the survival of irrational traders and contrast them with the partial equilibrium analysis. In Section 6, we examine the price impact of the irrational traders and how it affects their survival. Section 7 discusses the equilibrium portfolio policies and demonstrates ow the effects on price we describe extend beyond the first two moments and affect traders' portfolio policies and long-run survival. Section 8 concludes the paper with a short summary and some suggestions for future research. Appendix A provides additional results on the dynamics of wealth distribution and the welfare implications of irrational beliefs. All proofs are given in Appendix B.

\section{The Model}

The economic mechanisms that determine the survival of irrational traders and their impact on prices can be studied in a simple, parsimonious model, which is defined as follows.

\section{Information structure}

The economy has a finite horizon and evolves in continuous time. Uncertainty is described by a one-dimensional, standard Brownian motion $B_{t}$ for $0 \leq t \leq T$, defined on a complete probability space $(\Omega, F, P)$, where $F$ is the augmented filtration generated by $B_{t}$.

\section{Financial markets}

There is a single share of a risky asset in the economy, the stock, which is a claim on a terminal dividend payment $D_{T}$ at time $T . D_{T}$ is the value of a geometric Brownian motion $D_{t}$ at time $T$, where $D_{0}=1$ and

$$
d D_{t}=D_{t}\left(\mu d t+\sigma d B_{t}\right), \quad \sigma>0 .
$$

There is also a zero coupon bond available in zero net supply. Each unit of the bond makes

a certain payment of one at time $T$. We use the risk-free bond as the numeraire and denote the price of the stock at time $t$ by $S_{t}$. 


\section{Endowments}

There are two competitive traders in the economy, each endowed with a half share of the stock (and none of the bond) at time zero.

\section{Trading strategies}

Financial markets are frictionless, and there are no constraints on lending and borrowing. Traders' trading strategies satisfy the standard integrability condition used to avoid pathologies

$$
\int_{0}^{T} \theta_{t}^{2} d\langle S\rangle_{t}<\infty
$$

where $\theta_{t}$ is the number of shares of the stock held in the portfolio at time $t$ and $\langle S\rangle_{t}$ is the quadratic variation process of $S_{t}$.

\section{Preferences and beliefs}

Both traders have constant relative risk aversion utility, defined over their consumption at time $T$ :

$$
\frac{1}{1-\gamma} C_{T}^{1-\gamma}, \quad \gamma \geq 1 .
$$

For ease of exposition, we only consider the cases when $\gamma \geq 1$. The cases when $0<\gamma<1$ can be analyzed similarly and the results are similar in spirit.

Standard aggregation results imply that each trader in our model can actually represent a collection of traders with the same preferences. This provides a justification for our competitive assumption for each of the traders. The first trader, the rational trader, knows the true probability measure $P$ and maximizes expected utility

$$
\mathrm{E}_{0}^{P}\left[\frac{1}{1-\gamma} C_{r, T}^{1-\gamma}\right]
$$

where the subscript $r$ denotes quantities associated with the rational trader. The second trader, the irrational trader, believes incorrectly that the probability measure is $Q$, under which

$$
d B_{t}=(\sigma \eta) d t+d B_{t}^{Q}
$$


and hence

$$
d D_{t}=D_{t}\left[\left(\mu+\sigma^{2} \eta\right) d t+\sigma d B_{t}^{Q}\right]
$$

where $B_{t}^{Q}$ is the standard Brownian motion under the measure $Q$ and $\eta$ is a constant, parameterizing the degree of irrationality of the irrational trader. When $\eta$ is positive, the irrational trader is optimistic about the prospects of the economy and overestimates the rate of growth of the aggregate endowment. Conversely, a negative $\eta$ corresponds to a pessimistic irrational trader. The irrational trader maximizes expected utility using belief $Q$ :

$$
\mathrm{E}_{0}^{Q}\left[\frac{1}{1-\gamma} C_{n, T}^{1-\gamma}\right]
$$

where the subscript $n$ denotes quantities associated with the irrational trader.

Because $\eta$ is assumed to be constant, the probability measure of the irrational trader $Q$ is absolutely continuous with respect to the objective measure $P$, i.e., both traders agree on zero-probability events. Letting $\xi_{t} \equiv(d Q / d P)_{t}$ denote the density (Radon-Nikodym derivative) of the probability measure $Q$ with respect to $P$,

$$
\xi_{t}=e^{-\frac{1}{2} \eta^{2} \sigma^{2} t+\eta \sigma B_{t}}
$$

the irrational trader maximizes

$$
\mathrm{E}_{0}^{Q}\left[\frac{1}{1-\gamma} C_{n, T}^{1-\gamma}\right]=\mathrm{E}_{0}^{P}\left[\xi_{T} \frac{1}{1-\gamma} C_{n, T}^{1-\gamma}\right] .
$$

This permits us to interpret the objective of the irrational trader as the expected value of a state-dependent utility function, $\xi_{T} \frac{1}{1-\gamma} C_{n, T}^{1-\gamma}$, under the true probability measure $P$.

The equivalence between incorrect beliefs and state dependent preferences raises the conceptual question about the precise definition of irrationality. It is beyond the scope of this paper to address this question, and our analysis of this form of irrationality is primarily motivated by the fact that it is widely adopted in the recent literature on behavioral models of asset prices. 


\section{The Equilibrium}

The competitive equilibrium of the economy defined above can be solved analytically. Since there is only one source of uncertainty in the economy, the financial market is dynamically complete as long as the volatility of stock returns remains non-zero almost surely. Consequently, the equilibrium allocation is efficient and can be characterized as the solution to a central planner's problem:

$$
\begin{array}{ll}
\max & {\left[\frac{1}{1-\gamma} C_{r, T}^{1-\gamma}+b \xi_{T} \frac{1}{1-\gamma} C_{n, T}^{1-\gamma}\right]} \\
\text { s.t. } & C_{r, T}+C_{n, T}=D_{T}
\end{array}
$$

where $b$ is the ratio of utility weights. The equilibrium allocation is characterized in the following proposition.

Proposition 1 For the economy defined in Section 2, the equilibrium allocation between the two traders is

$$
\begin{aligned}
C_{r, T} & =\frac{1}{1+\left(b \xi_{T}\right)^{1 / \gamma}} D_{T} \\
C_{n, T} & =\frac{\left(b \xi_{T}\right)^{1 / \gamma}}{1+\left(b \xi_{T}\right)^{1 / \gamma}} D_{T}
\end{aligned}
$$

where

$$
b=e^{(\gamma-1) \eta \sigma^{2} T}
$$

The price of a financial security with the terminal payoff $Z_{T}$ is given by

$$
P_{t}=\frac{\mathrm{E}_{\mathrm{t}}\left[\left(1+\left(b \xi_{T}\right)^{1 / \gamma}\right)^{\gamma} D_{T}^{-\gamma} Z_{T}\right]}{\mathrm{E}_{\mathrm{t}}\left[\left(1+\left(b \xi_{T}\right)^{1 / \gamma}\right)^{\gamma} D_{T}^{-\gamma}\right]}
$$

For the stock, $Z_{T}=D_{T}$ and its return volatility is bounded between $\sigma$ and $\sigma(1+|\eta|)$.

Since the instantaneous volatility of stock returns is bounded below by $\sigma$, the stock and the bond dynamically complete the financial market. In the limiting cases when only the rational or the irrational trader is present, the stock prices, denoted by $S_{r, t}$ and $S_{n, t}$, 
respectively, are given by

$$
\begin{aligned}
S_{r, t} & =e^{\left(\mu / \sigma^{2}-\gamma\right) \sigma^{2} T+\frac{1}{2}(2 \gamma-1) \sigma^{2} t+\sigma B_{t}} \\
S_{n, t} & =e^{\left(\mu / \sigma^{2}-\gamma+\eta\right) \sigma^{2} T+\frac{1}{2}[(2 \gamma-1)-2 \eta] \sigma^{2} t+\sigma B_{t}}=S_{r, t} e^{\eta \sigma^{2}(T-t)} .
\end{aligned}
$$

We will use this equilibrium model to analyze the survival and extinction of the traders. We employ two definitions of extinction, and, conversely, of survival.

Definition 1 The irrational trader is said to experience relative extinction in the long-run if

$$
\lim _{T \rightarrow \infty} \frac{C_{n, T}}{C_{r, T}}=0 \quad \text { a.s. }
$$

The relative extinction of the rational trader can be defined symmetrically. A trader is said to survive relatively in the long-run if relative extinction does not occur.

Definition 2 The irrational trader is said to experience long-run extinction in the absolute sense if

$$
\lim _{T \rightarrow \infty} C_{n, T}=0 \quad \text { a.s. }
$$

The absolute extinction of the rational trader can be defined symmetrically. A trader is said to survive absolutely in the long-run if absolute extinction does not occur.

In the above definitions and throughout the paper all limits are understood to be almost sure (under the true probability measure $P$ ) unless specifically stated otherwise.

Clearly, relative survival is sufficient for absolute survival but as our analysis demonstrates, absolute survival is not sufficient for relative survival. Similarly, absolute extinction implies relative extinction, but the converse is not true. Throughout this paper, we use the notion of relative extinction or survival unless it is specified otherwise.

In our model, the final wealth of each trader equals the terminal consumption. Thus, the definitions of survival and extinction are equivalent to a similar definition using wealth. 


\section{A Special Case: Logarithmic Preferences}

We begin analyzing the equilibrium by considering a special case of the economy where all traders have logarithmic preferences. When both the rational and irrational traders have logarithmic preferences, we have the following result:

Proposition 2 Suppose $\eta \neq 0$. For $\gamma=1$, the irrational trader never survives.

This result is immediate. For $\gamma=1$, the rational trader holds the portfolio with maximum expected growth. Any deviation in beliefs from the true probability causes the irrational trader to move away from the maximum growth portfolio, which leads to his long-run relative extinction.

Our interest, however, is not merely on the survival of the irrational trader, but also on the impact of irrationality on the long-run stock price. Under logarithmic preferences, $b=1$ and from Proposition 2 the stock price is

$$
S_{t}=\frac{1+\xi_{t}}{\mathrm{E}_{\mathrm{t}}\left[\left(1+\xi_{T}\right) / D_{T}\right]}=\frac{1+\xi_{t}}{1+e^{-\eta \sigma^{2}(T-t)} \xi_{t}} \frac{1}{e^{\left(-\mu+\frac{1}{2} \sigma^{2}\right) T+\frac{1}{2} \sigma^{2}(T-t)-\sigma B_{t}}} .
$$

Moreover, the moments of stock returns can be derived explicitly. Let $\alpha_{t}$ denote the relative wealth of the rational trader,

$$
\alpha_{t} \equiv \frac{W_{r, t}}{W_{r, t}+W_{n, t}}
$$

the conditional volatility of stock returns is given by

$$
\sigma_{S, t}=\sigma+\eta \sigma\left(1-\alpha_{t}\right)-\eta \sigma\left[1-\frac{1}{1+e^{-\eta \sigma^{2}(T-t)}\left(\alpha_{t}^{-1}-1\right)}\right]
$$

while the conditional mean is

$$
\mu_{S, t}=\sigma_{S, t}^{2}-\eta \sigma\left(1-\alpha_{t}\right) \sigma_{S, t}
$$

The presence of the irrationality parameter $\eta$ in the expressions for the stock price and its first two moments provides our first demonstration that the irrational trader can maintain influence on the stock price for long periods of time even though he does not survive in the long run. 
This can be seen explicitly from a numerical illustration with parameters corresponding to an economy with a relatively long horizon. The irrational trader is assumed to be pessimistic $(\eta=-4)$. The horizon of the economy is set at $T=400$. We let the current time $t$ be sufficiently large, so with high probability most of wealth in the economy is controlled by the rational trader. For convenience, we define the following normalized state variable:

$$
g_{s, t} \equiv \frac{B_{t}-B_{s}}{\sqrt{t-s}} .
$$

It is easy to show that $g_{s, t}$ is the unanticipated dividend growth normalized by its standard deviation, which has a standard normal distribution. Figure 1 plots the stock price volatility and the wealth distribution at $t=100$ against the normalized state variable $g_{0, t}$. The probability density for $g_{0, t}$ is illustrated by the shaded area (with the vertical axis on the right). The bottom panel of Figure 1 shows that with almost probability one, the wealth of the economy is all controlled by the rational trader at this time. Yet as the top panel of the figure shows, the conditional volatility of stock returns is very different from $\sigma$, the volatility of the state variable $D_{t}$, which would be its value in the economy populated by only one type of traders. In particular, over a large range of values of the dividends, the conditional volatility of returns is approximately equal to $\sigma(1-\eta) \neq \sigma$.

To examine the long-run stock price more generally we employ asymptotic analysis. We call two stochastic processes asymptotically equivalent if for large values of $T$, their ratio converges to one. That is,

Definition 3 (Asymptotic Equivalence) Two stochastic processes, $X_{t}$ and $Y_{t}$ are said to be asymptotically equivalent if

$$
\lim _{T \rightarrow \infty} \frac{X_{T}}{Y_{T}}=1 \quad \text { a.s. }
$$

which are denoted by $X_{T} \sim Y_{T}$.

As the horizon of the economy, $T$, increases, we need to have some sense of what it means for a particular property of the economy to persist for a significant period of time. Suppose, for example, we say that the impact of the irrational trader on a variable is significant as long as the variable exceeds a fixed level $e$ within a time interval. If as $T$ grows, this time interval also grows with $T$ but at a slower rate, this would mislead us into arguing that the impact of the irrational trader was significant, even though it was not persistent. Persistence requires that the proportion of time over which the impact is significant does not go to zero 


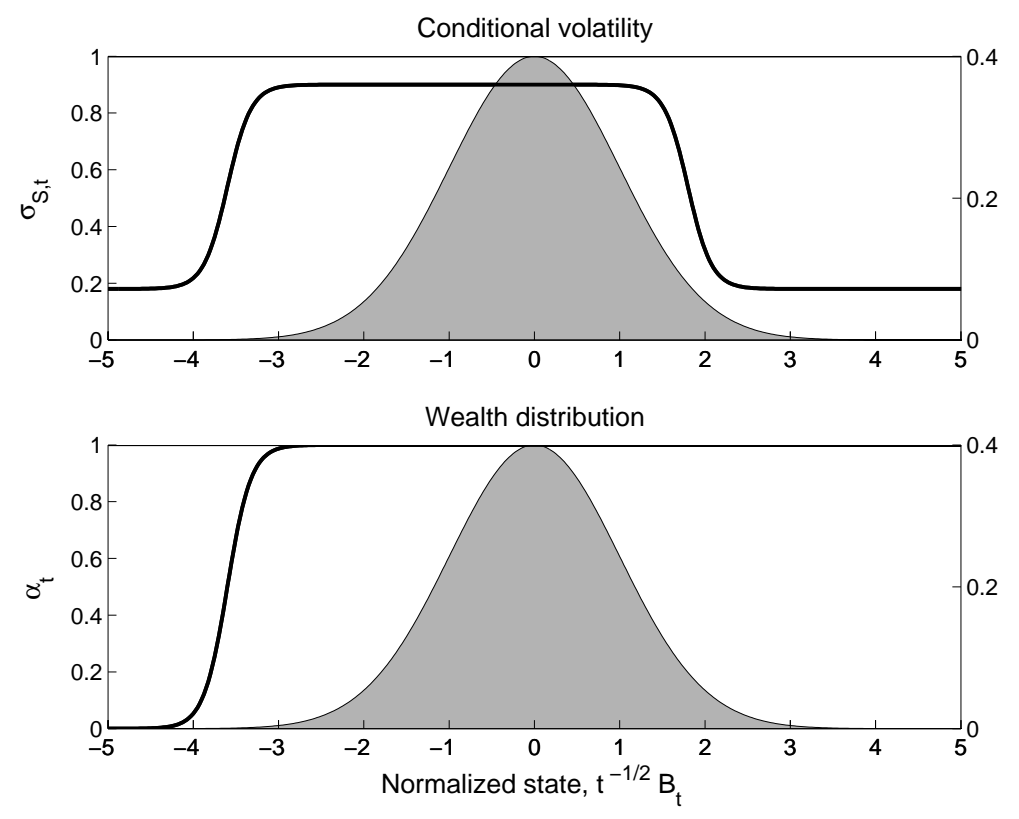

Figure 1: The conditional volatility of stock returns, $\sigma_{S, t}$ and the wealth distribution $\alpha_{t}=$ $W_{r, t} /\left(W_{r, t}+W_{n, t}\right)$ are plotted against the normalized state variable, $g_{0, t} \equiv B_{t} / \sqrt{t}$. The shaded area is the probability density function of the normalized state variable (vertical axis on the right). The model parameters are set at $\mu=0.12, \sigma=0.18, \eta=-4, T=400$ and $\gamma=1$. The current time is $t=100$.

as $T$ grows. Conversely, if the proportional amount of time over which there is negligible impact is going to one, then we conclude that the irrational trader has no persistent impact.

To make this more formal, we will consider a sequence of economies with increasing horizon $T$, where the current time of observation is chosen to be $t=\lambda T, 0<\lambda \leq 1$. As the horizon of the economy approaches infinity, the "current" time $t$ increases as well, but it remains at a constant fraction of the horizon of the economy. Moreover, the time remaining until the final date of the economy is also increasing proportionally to $T$. Since the properties of the equilibrium prices and quantities depend on how much time is remaining until the final date, they depend on $\lambda$.

We have the following result on the persistence of the price impact of irrationality when traders have logarithmic preferences:

Proposition 3 Suppose $\gamma=1$ and $\eta<0$. Let $\lambda_{S}=2 /(2-\eta)$. At $t=\lambda T$, the stock price 
behaves as follows when $T \rightarrow \infty$ :

$$
S_{t} \sim \begin{cases}S_{r, t} e^{\eta\left[\sigma^{2} T+\left(\frac{1}{2} \eta-1\right) \sigma^{2} t-\sigma B_{t}\right]}, & 0<\lambda<\lambda_{S} \\ S_{r, t}, & \lambda_{S}<\lambda \leq 1\end{cases}
$$

where $S_{r, t}$, defined in Equation (13) with $\gamma=1$, is the stock price when only the rational trader is present. The limiting values of the instantaneous moments of stock returns are equal to the moments of the corresponding asymptotic expression (18).

It follows from Proposition 3 that for any $t<\lambda_{S} T$, the price influence of the irrational trader remains significant, that is, $S_{t} \neq S_{r, t}$, despite the fact that as $T$ approaches infinity, $t$ can be arbitrarily large and the wealth share of the irrational trader becomes negligible.
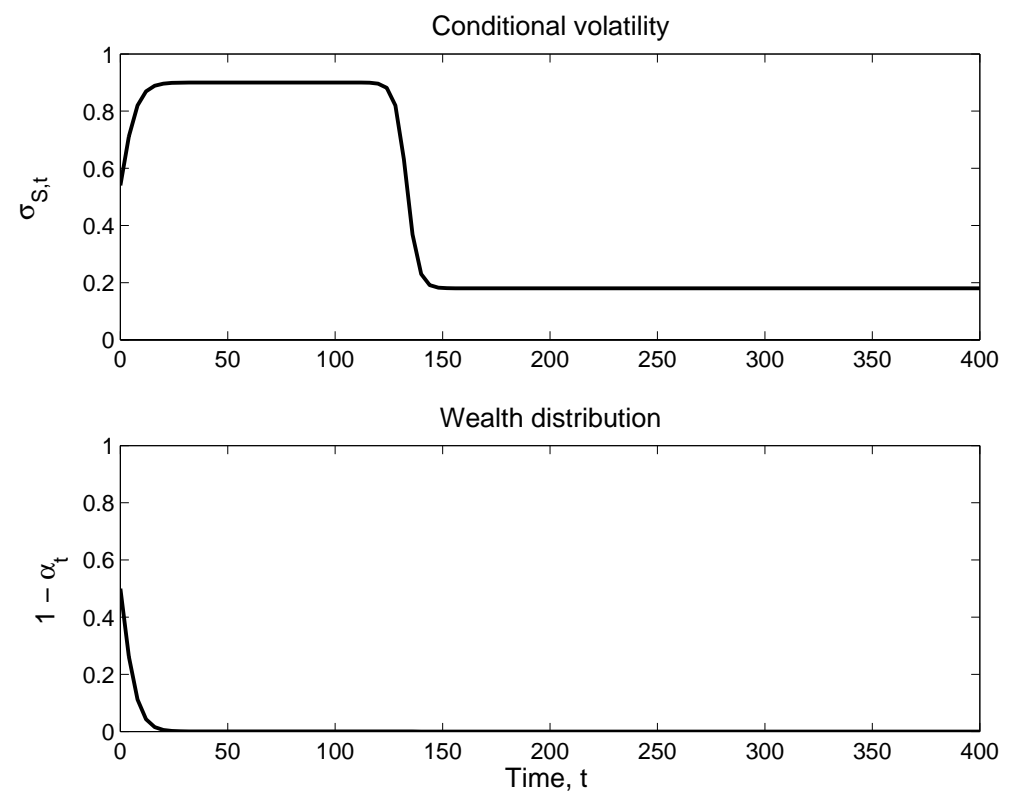

Figure 2: The maximum likelihood path of the irrational trader's wealth share, $1-\alpha_{t}$, and the stock price volatility, $\sigma_{S, t}$. The model parameters are set at $\mu=0.12, \sigma=0.18, \eta=-4, T=400$ and $\gamma=1$.

What Propositions 3 describes is the situation where the wealth share of the irrational trader and his price impact converge to zero over time at drastically different rates. Figure 2 illustrates the most likely path over time (the path with highest probability) for the irrational trader's wealth share and the stock price volatility. In fact, his wealth share diminishes to zero exponentially while his price impact diminishes at a much slower rate. The stock price volatility stays away from its asymptotic level for an extended period of time before eventually converging to its limiting value. 
In order to better understand how the irrational trader can exert influence on the stock price despite having negligible wealth, we examine how his portfolio policy affects the state price density (SPD). With the horizon of the economy set at $T=30$, the left panels of Figure 3 plot the relative consumption shares of the rational and the irrational traders at two different times, $t=0,20$, as a function of the normalized state variable, $g_{t, T}$ (i.e., the normalized unanticipated dividend growth from $t$ to $T$ defined in (17)). At each date, the state of the economy is conditioned on $B_{t}=0$, the most likely state. For $t=0$, the irrational trader owns half of the economy. But at $\eta=-4$, he is very pessimistic and bets on states of low dividends (states toward the left end of the horizontal axis). This is shown in the top left panel of Figure 3. The dashed line plots his terminal consumption for different states of the economy. Over time, these 'bad' states become even less likely and the irrational trader's bets become less valuable. Thus, his wealth decreases. At $t=20$ and $B_{t}=0$, these bad states become extremely unlikely and the irrational trader has lost most of his wealth. His wealth relative to the wealth of the rational trader has reduced from 1 at $t=0$ to 0.0056 . As shown in the bottom left panel of Figure 3, going forward, he consumes a non-trivial fraction of the total wealth only in the extreme states toward the left end of the horizontal axis. The probability of these states, as shown by the shaded area, becomes very small. So is the irrational trader's wealth.

In the two panels on the right of Figure 3, we plot the state price density against the normalized state variable $d_{T}$ at the two times, $t=0,20$, conditioned again on $B_{t}=0$. With logarithmic preferences, the equilibrium state price density at time $t$ is given by

$$
\phi_{t} \equiv \frac{\left(1+\xi_{T}\right) D_{T}^{-1}}{\mathrm{E}_{\mathrm{t}}\left[\left(1+\xi_{T}\right) D_{T}^{-1}\right]}
$$

which is represented by the dashed line in each of the two panels. The solid line plots the state price density when the economy is populated only by the rational traders, which can be obtained by setting $\xi_{T}=0$ in the above expression for $\phi_{t}$. The top panel gives the state price density at $t=0$. It is obvious that at this point, the irrational trader owns half of the total wealth and his portfolio policy has a significant influence on the state price density over the whole range. In particular, being pessimistic, he is effectively betting on the bad states, which causes the state price density to increase for the bad states and decrease for the good states. This is shown by the difference between the dashed line, the state price density in the presence of the irrational trader, and the solid line, the state price density without the irrational trader. As time passes, his wealth dwindles and his influence on the state price density diminishes quickly for most of the states, as the bottom panel for $t=20$ 

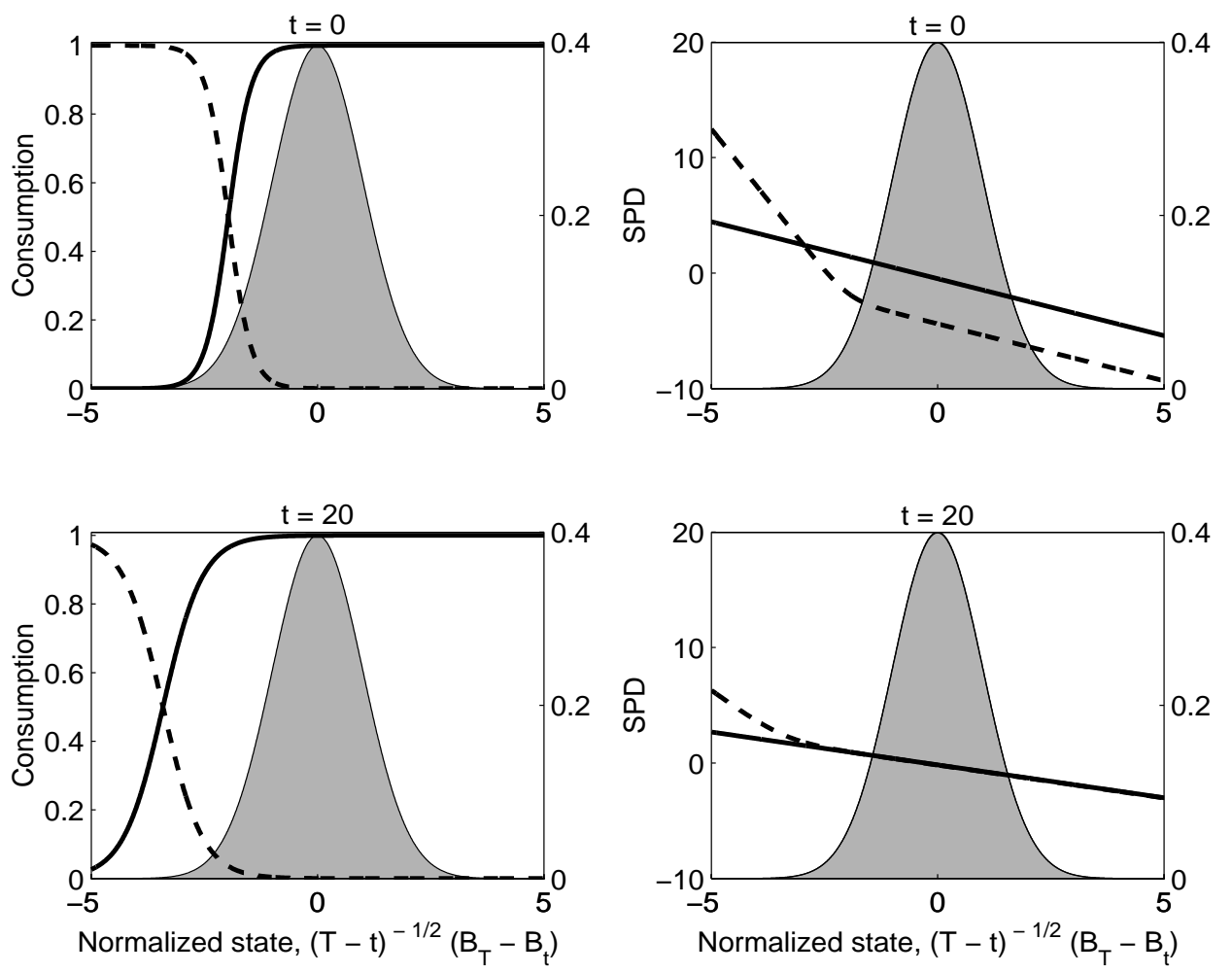

Figure 3: The terminal consumption of the rational and irrational traders as a fraction of the total consumption and the state price density (SPD) in different terminal states of the economy at different times. The model parameters are set to be $\mu=0.12, \sigma=0.18, \eta=-4, \gamma=1$ and $T=30$. The horizontal axis in all panels is the normalized state variable $g_{t, T} \equiv\left(B_{T}-B_{t}\right) / \sqrt{T-t}$, which has a standard normal distribution with zero mean and unit variance, which is shown by the shaded area (vertical axis on the right). In the two panels on the left, the terminal consumption for the rational trader (the solid line) and the irrational trader (the dotted line) are plotted against the normalized state variable at times $t=0,20$, respectively, when $B_{t}=0$. In the two panels on the right, the dashed line plots the logarithm of the state price density at times $t=0,20$, respectively, which is $\ln \left\{\left[\left(1+\xi_{T}\right) / D_{T}\right] / \mathrm{E}_{\mathrm{t}}\left[\left(1+\xi_{T}\right) / D_{T}\right]\right\}$. The solid line plots the logarithm of the state price density in the economy populated only by the rational traders, which is $\ln \left\{D_{T}^{-1} / \mathrm{E}_{\mathrm{t}}\left[D_{T}^{-1}\right]\right\}$.

shows. However, for the extremely bad states, his influence remains significant because he is still betting heavily on these states.

In order to further examine how the irrational trader can affect the state-price density for the bad states, let us consider the following thought experiment. We start with the economy inhabited only by the rational trader. The state price density, denoted by $\phi$, is proportional to the rational trader's marginal utility $u^{\prime}(C): \phi=a u^{\prime}(C)$, where $a$ is a constant. Suppose now the irrational trader is introduced into the economy and he purchases $\delta$ units of state-contingent claims that pay off only when the aggregate consumption is $C$. The total cost is $w \equiv \phi \delta \approx a u^{\prime}(C) \delta$ when $\delta$ is small. The state-price density now will change 
by $\Delta \phi \approx-a u^{\prime \prime}(C) \delta$. Divided by the wealth spent by the irrational trader, we obtain the marginal change in the state-price density:

$$
\frac{\Delta \phi}{w}=\frac{u^{\prime \prime}(C)}{u^{\prime}(C)}=\frac{1}{C}
$$

which is independent of $\delta$. Clearly, for the bad states when $C$ is low (close to zero), the irrational trader can have a large impact on the state-price density with little wealth if he decides to bet on these states. Moreover, through his impact on the state-price density in bad states, the irrational trader can influence asset prices, such as the prices of the stock and the bond. Given that the bond is used as a numeraire and its price is always one, this influence is captured in the stock price. In particular, we have

$$
S_{t}=\mathrm{E}_{\mathrm{t}}\left[\phi_{t} D_{t}\right]
$$

which is computed in Equation (16). As $t$ increases, Equation (18) gives the stock price asymptotically. The above analysis shows that despite the low probability and low dividends of the extreme bad states, the irrational trader can maintain his influence on the stock price through his impact on the state price density of these states, even as his wealth diminishes over time.

The simple case of logarithmic preferences clearly shows that survival and price impact are in general not equivalent. In particular, survival is not a necessary condition for the irrational traders to influence long-run prices, and depending on their beliefs, irrational traders can maintain a significant price impact even as their wealth becomes negligible over time.

\section{The Survival of Irrational Traders}

In the case of logarithmic preferences, the irrational trader does not survive in the long-run simply because his portfolio grows more slowly than the maximum growth rate which is achieved by the rational trader. For relative risk aversion different from one, though, the rational trader no longer holds the optimal growth portfolio and under an incorrect belief, the irrational trader may end up holding a portfolio that is closer to the optimal growth portfolio, and so his wealth may grow more rapidly. This was the argument put forward by DSSW using a partial equilibrium setting similar to ours. In this section, we examine the long-run survival of the irrational trader in a general equilibrium setting. 


\subsection{Heuristic Partial Equilibrium Analysis}

We first follow the partial equilibrium argument of DSSW and derive conditions under which the irrational trader may survive in the long-run despite having incorrect beliefs. The essence of the partial equilibrium argument is to examine a limiting case in which one of the two traders controls most of the aggregate wealth in the economy while the other trader is infinitesimal. The argument then assumes that the infinitesimal agent has no impact on market prices and computes the implied growth rate of both traders' wealth. If the wealth of the infinitesimally small trader has a higher growth rate, and therefore the share of wealth of such a trader is growing over time, then DSSW conclude that such a trader would be able to successfully "invade" the economy and hence must survive in the long-run, "in the sense that their wealth share does not drop toward zero in the long run with probability one."

Assume first that the rational trader dominates the economy and the prices of financial assets are as if there were no irrational traders. Let $\mu_{S}$ and $\sigma_{S}$ denote the drift and volatility of the stock price process:

$$
d S_{t}=S_{t}\left(\mu_{S} d t+\sigma_{S} d B_{t}\right)
$$

It is easy to show that

$$
\mu_{S}=\gamma \sigma^{2}, \quad \sigma_{S}=\sigma
$$

The rational trader invests only in the stock. The rate of growth of the rational trader's portfolio, defined as the drift of the logarithm of the trader's wealth, is given by

$$
\mu_{S}-\frac{1}{2} \sigma_{S}^{2}=\frac{1}{2}(2 \gamma-1) \sigma^{2}
$$

Under the beliefs of the irrational trader (under the measure $Q$ ), the drift of the stock price process is

$$
\widehat{\mu}_{S}=\mu_{S}+\sigma^{2} \eta
$$

and the volatility remains $\sigma$. The irrational trader invests a fraction $w_{n}=\widehat{\mu}_{S} /\left(\gamma \sigma^{2}\right)=1+\eta / \gamma$ of his wealth in the stock. Thus, the growth rate of the irrational trader's portfolio is

$$
\mu_{S}-\frac{1}{2} \sigma^{2}+\frac{1}{2} \frac{\sigma^{2}}{\gamma^{2}} \eta\left(\gamma \eta^{\star}-\eta\right)
$$


where we define

$$
\eta^{\star} \equiv 2(\gamma-1)
$$

The growth rate of wealth of the "invading" irrational trader is higher than that of the dominant rational trader if and only if $0<\eta<\gamma \eta^{\star}$.

Next, assume that the irrational trader dominates the economy. Repeating the steps of the previous analysis, the volatility of the stock price remains at $\sigma$ and the drift becomes

$$
\mu_{S}=\gamma \sigma^{2}-\eta \sigma^{2}
$$

For the irrational trader, the growth rate of the portfolio is

$$
\mu_{S}-\frac{1}{2} \sigma^{2}
$$

while for the rational trader it is

$$
\mu_{S}-\frac{1}{2} \sigma^{2}+\frac{1}{2} \frac{\sigma^{2}}{\gamma^{2}}(2 \gamma-1) \eta\left(\eta-\frac{\gamma}{2 \gamma-1} \eta^{\star}\right)
$$

The rational trader's portfolio grows faster than the irrational trader's portfolio if and only if $\eta<0$ or $\eta>\frac{\gamma}{2 \gamma-1} \eta^{\star}$.

The partial equilibrium analysis thus appears to provide sufficient conditions for long-run survival of both types of traders. In particular, for $\gamma>1$

$$
\begin{array}{lll}
0<\eta<\frac{\gamma}{2 \gamma-1} \eta^{\star} & \Rightarrow \text { Irrational trader survives } \\
\frac{\gamma}{2 \gamma-1} \eta^{\star}<\eta<\gamma \eta^{\star} & \Rightarrow \text { Both traders survive } \\
\eta<0 \text { or } \eta>\gamma \eta^{\star} & \Rightarrow \text { Rational trader survives }
\end{array}
$$

For $\gamma=1$, only the rational trader survives regardless of the value of $\eta$. These results are summarized in the phase diagram shown in Figure 4. Since $\gamma /(2 \gamma-1) \leq 1$ for $\gamma \geq 1, \eta^{\star}$ belongs to the second region defined in (20).

\subsection{General Equilibrium Analysis}

The partial equilibrium analysis seems straightforward and intuitive. However, it is based on the assumption that the stock price is unaffected by the trader whose wealth approaches 


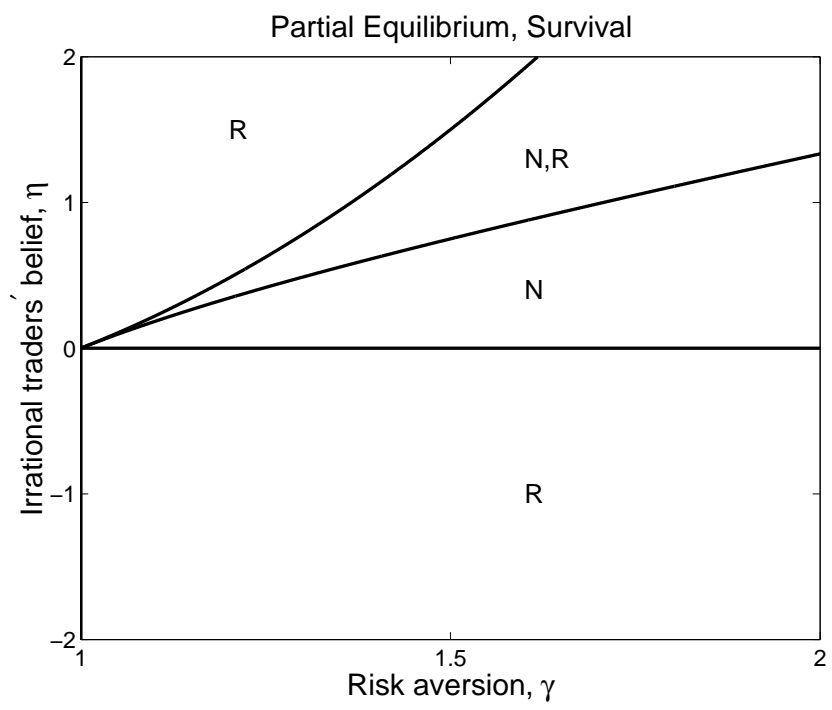

Figure 4: The survival of rational and irrational traders for different values of $\eta$ and $\gamma$ in partial equilibrium. For each region in the parameter space, we document which of the agents survives in the long run. "R" means that survival of the rational trader is guaranteed inside the region, "N" corresponds to the irrational trader, "N,R" means that both traders survive.

zero in the long run. The fact that this assumption does not hold in the case of logarithmic preferences suggests that the partial equilibrium analysis cannot, in general, be trusted to correctly analyze survival and extinction. From the competitive equilibrium derived in Section 3, we have the following result:

Proposition 4 Suppose $\eta \neq 0$. For $\gamma=1$, the irrational trader never survives. For $\gamma>1$, only one of the traders survives in the long run. In particular,

Pessimistic irrational trader: $\eta<0 \quad \Rightarrow$ Rational trader survives

Moderately optimistic irrational trader: $0<\eta<\eta^{\star} \Rightarrow$ Irrational trader survives

Strongly optimistic irrational trader: $\eta>\eta^{\star} \quad \Rightarrow$ Rational trader survives

where $\eta^{\star}=2(\gamma-1)$. For $\eta=\eta^{\star}$, both rational and irrational traders survive.

For $\gamma>1$, Proposition 4 identifies three distinct regions in the parameter space as shown in Figure 5. For $\eta<0$, the irrational trader is pessimistic and does not survive in the long-run. For $0<\eta<\eta^{\star}$, the irrational trader is moderately optimistic and survives in the long-run while the rational trader does not. For $\eta>\eta^{\star}$, the irrational trader is strongly optimistic and does not survive. 


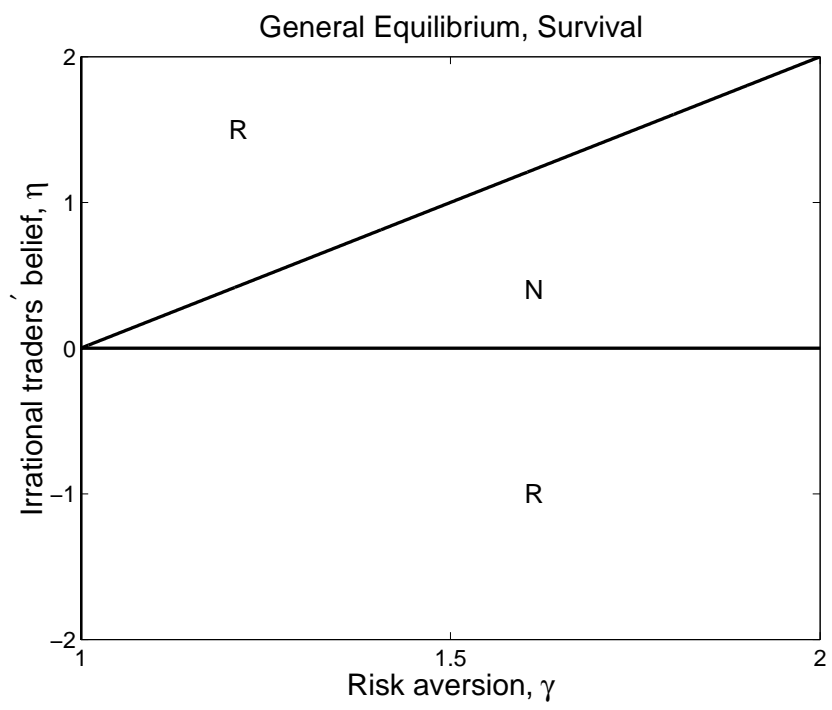

Figure 5: The survival of rational and irrational traders for different values of $\eta$ and $\gamma$ in general equilibrium. For each region in the parameter space, we document which of the agents survives in the long run. "R" means that survival of the rational trader is guaranteed inside the region, "N" corresponds to the irrational trader.

By contrast with the partial equilibrium displayed in Figure 4 where there is a region in which both traders survive, other than the knife-edge case $\left(\eta=\eta^{\star}\right)$, only one of the traders can survive in general equilibrium. Furthermore, the surviving trader eventually dominates the economy and owns most of the wealth. In general, however, survival and dominance may not be equivalent. In addition, comparing Figures 4 and 5 we see that for some regions of parameter values, the partial equilibrium argument leads to incorrect predictions about the survival of traders. In particular, the general equilibrium divides the mixed partial equilibrium where both traders seem to survive into two sections; a lower one below $\eta^{\star}$ where only the irrational trader survives and an upper one in which only the rational trader survives.

In order to gain more insight into what determines the survival of the irrational trader, we examine the terminal wealth (consumption) profiles of the rational and irrational traders. The two panels on the left in Figure 6 show the two traders' terminal wealth profiles for two values of $T$ (10 and 30) when the irrational trader is pessimistic. The solid line shows the terminal wealth share of the rational trader and the dashed line shows that for the irrational trader. As expected, the rational trader ends up with more wealth in good states of the economy (when the dividend is high) while the irrational trader, being pessimistic, ends up with more wealth in the bad states of the economy. As the horizon increases, the irrational 

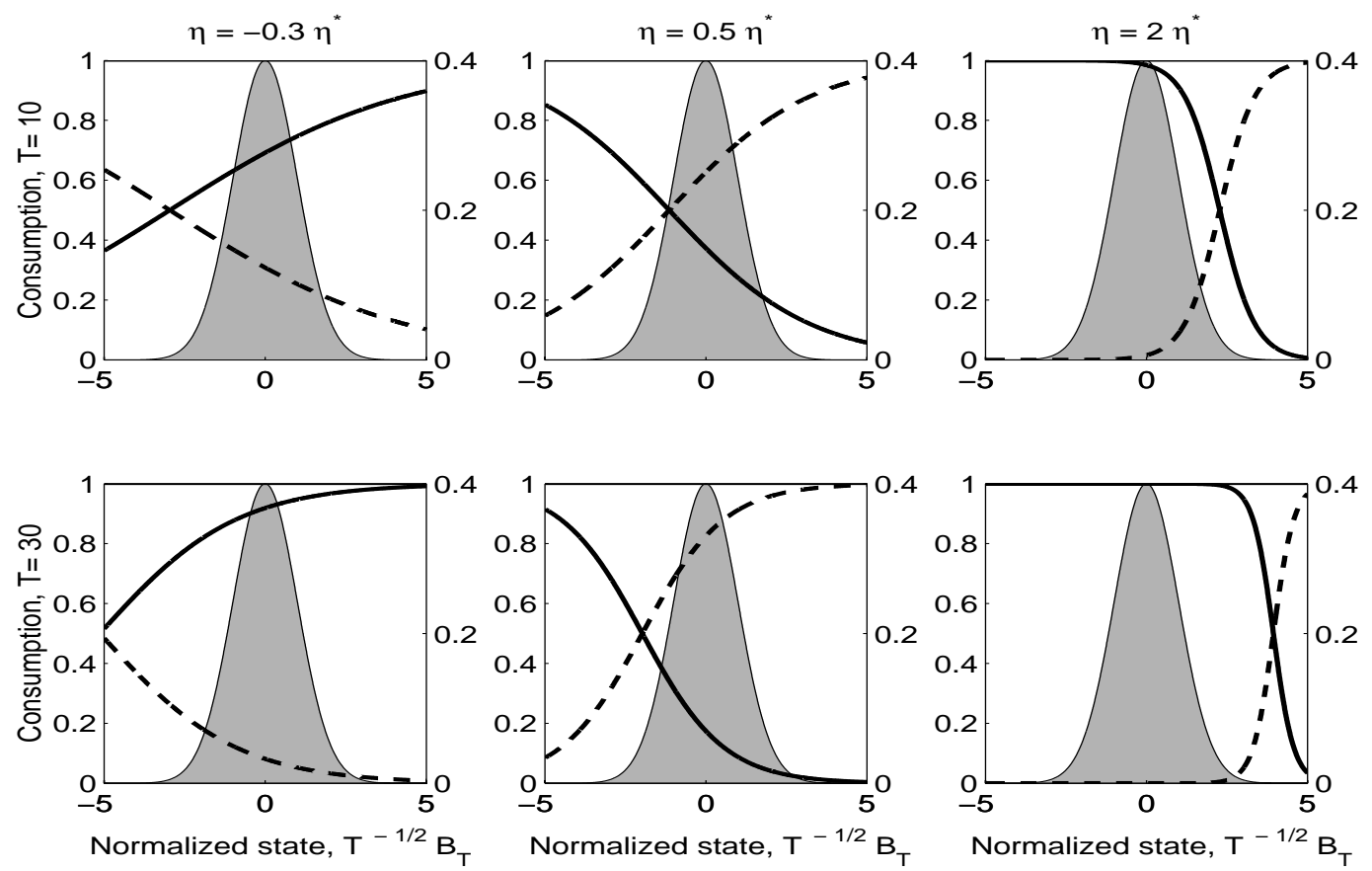

Figure 6: The terminal consumption of rational and irrational traders for different horizon $T$. We consider two values of $T, 10$ and 30, respectively. The model parameters are set at $\mu=0.12$, $\sigma=0.18$, and $\gamma=5$. We consider three distinctive cases for the irrational trader's belief: (1) pessimistic, $\eta=-0.3 \eta^{\star}$, (2) moderately optimistic, $\eta=0.5 \eta^{\star}$, and (3) strongly optimistic, $\eta=2 \eta^{\star}$. The horizontal axis in all panels is the normalized state variable $g_{0, T} \equiv\left(B_{T}\right) / \sqrt{T}$, which has a standard normal distribution with zero mean and unit variance, which is shown by the shaded area (vertical axis on the right). The two panels on the left show the terminal consumption, as a fraction of the total consumption, of the rational trader (solid line) and the irrational trader (dashed line) with a pessimistic belief, i.e., $C_{r, T} / D_{T}$ and $C_{n, T} / D_{T}$, for the two values of the horizon, $T=10,30$, respectively. The two panels in the middle and on the right show the terminal consumption, as a fraction of the total consumption, of the rational trader and the irrational trader with a moderately and strongly optimistic beliefs for the two values of $T$, respectively.

trader ends up with non-trivial wealth in more extreme and less likely, low dividend states. When the irrational trader is mildly optimistic, the situation is different. His impact on the prices make the bad states (i.e., the low dividend states) cheaper than the good states. This induces the rational trader to accumulate more wealth in the bad states by giving up wealth in the good states, including those with high probabilities. As a result, the irrational trader is more likely to end up with more wealth. When strongly optimistic, the irrational trader ends up accumulating wealth in very unlikely, good states by giving up wealth in most other states, which leads to his extinction in the long-run. 


\subsection{Absolute Extinction}

In the above discussion, we used the notion of extinction in the relative sense that the relative wealth of a trader goes to zero in the long-run. Our equilibrium analysis gives the following result about absolute survival:

Proposition 5 Assume that the aggregate endowment is growing, i.e., $\mu>\frac{1}{2} \sigma^{2}$.

(i) The irrational trader experiences long-run absolute extinction if

$$
\eta<(\gamma-1)-\sqrt{(\gamma-1)^{2}+\gamma\left(2 \mu / \sigma^{2}-1\right)}
$$

or

$$
\eta>(\gamma-1)+\sqrt{(\gamma-1)^{2}+\gamma\left(2 \mu / \sigma^{2}-1\right)} .
$$

(ii) The rational trader experience long-run absolute extinction if $(\gamma-1)^{2}-\gamma\left(2 \mu / \sigma^{2}-1\right)>0$ and

$$
(\gamma-1)-\sqrt{(\gamma-1)^{2}-\gamma\left(2 \mu / \sigma^{2}-1\right)}<\eta<(\gamma-1)+\sqrt{(\gamma-1)^{2}-\gamma\left(2 \mu / \sigma^{2}-1\right)}
$$

Figure 7 shows the ranges of $\eta$, given $\gamma$, for the relative and absolute extinction of the two traders. This verifies that there are regions where the two concepts do not coincide. It is worth pointing out that for $\eta<(\gamma-1)-\sqrt{(\gamma-1)^{2}+\gamma\left(2 \mu / \sigma^{2}-1\right)}$, the irrational trader experiences absolute extinction and yet he can still have non-trivial price impact in the long-run (when $\eta<0$ ) as our discussion in the logarithmic case shows.

\section{The Price Impact of Irrational Traders}

The difference in the results between the partial equilibrium argument and the general equilibrium analysis on the irrational trader's survival comes from the fact that the price impact of irrational traders is taken into account in general equilibrium and ignored in partial equilibrium. The partial equilibrium "invasion" analysis of an infinitesimal trader, i.e., a trader who controls only a negligible fraction of the total wealth, relies on the following two assumptions: (i) such a trader has no impact on prices, (ii) the portfolio policies of both 


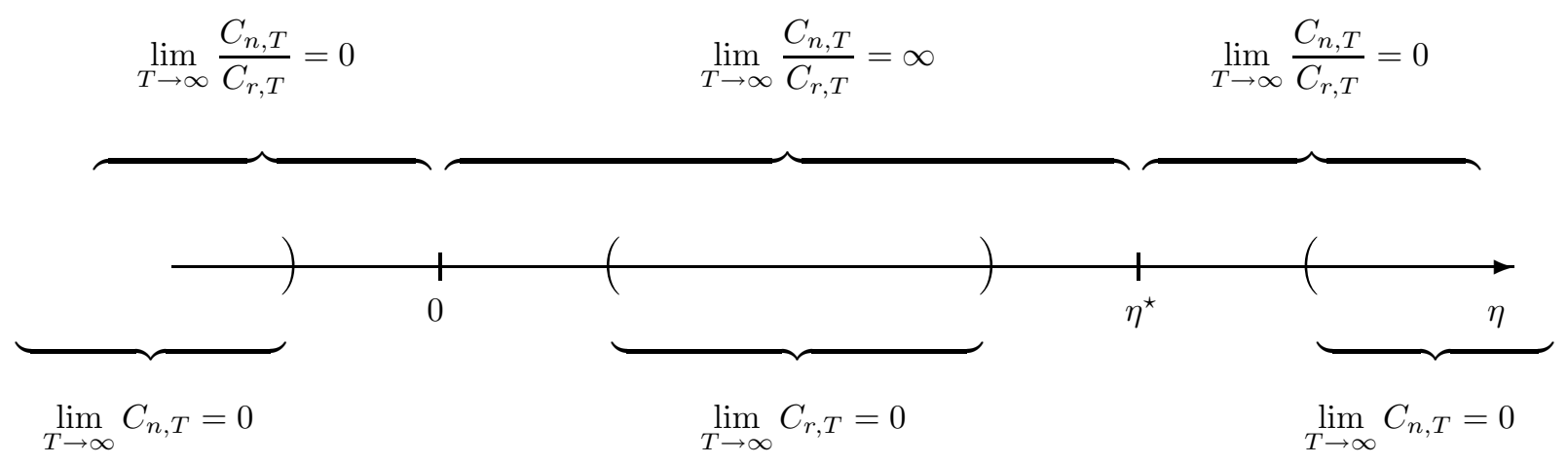

Figure 7: Comparison between relative extinction and absolute extinction.

traders are the same as if the prices are exclusively set by the dominant trader. Under these assumptions, the infinitesimal trader's wealth is growing as a fraction of the total and one can conclude that such an trader must survive in the long run.

Having derived the exact solution to the general equilibrium economy, we now investigate the validity of these two assumptions and characterize the precise combinations of the model parameters under which they fail to hold. We have already seen that in the case of logarithmic preferences, assumption (i) does not always hold. In this section we examine the validity of the first assumption in the general case for $\gamma>1$ and in Section 7 we examine the second assumption.

Our focus is on relative survival and, as in Section 4 when considering a sequence of economies with increasing horizon, we choose the time of observation to be $t=\lambda T, 0<$ $\lambda \leq 1$. We define three values of $\lambda$ to help us characterize points of change in the limiting behavior:

$$
\lambda_{S} \equiv \frac{2}{2 \gamma-\eta}, \quad \lambda_{r} \equiv \frac{\eta}{(\gamma-1)(2 \gamma-\eta)}, \quad \lambda_{n} \equiv \frac{\eta}{\eta(\gamma+1)-2 \gamma(\gamma-1)} .
$$

It is easy to verify that for $\eta<\eta^{\star}, 0<\lambda_{S} \leq 1$; for $0<\eta \leq \eta^{\star}, 0<\lambda_{r} \leq 1$; and for $\eta<0$ or $\eta>\eta^{\star}, 0<\lambda_{n} \leq 1$.

The limiting behavior of the stock price process can be characterized as follows.

Proposition 6 (Stock Price) At $t=\lambda T$, the stock price behaves as follows when $T \rightarrow \infty$. 
Case 1. Pessimistic Irrational Trader $(\eta<0)$ :

$$
S_{t} \sim \begin{cases}S_{r, t} e^{\eta\left[\sigma^{2} T+\frac{1}{2}(\eta-2 \gamma) \sigma^{2} t-\sigma B_{t}\right]}, & 0<\lambda<\lambda_{S} \\ S_{r, t}, & \lambda_{S}<\lambda \leq 1\end{cases}
$$

Case 2. Moderately Optimistic Irrational Trader $\left(0<\eta<\eta^{\star}\right)$ :

$$
S_{t} \sim \begin{cases}S_{n, t} e^{-\eta\left[\sigma^{2} T+\frac{1}{2}(\eta-2 \gamma+2) \sigma^{2} t-\sigma B_{t}\right]}, & 0<\lambda<\lambda_{S} \\ S_{n, t}, & \lambda_{S}<\lambda \leq 1\end{cases}
$$

Case 3. Strongly Optimistic Irrational Trader $\left(\eta^{\star}<\eta\right)$ :

$$
S_{t} \sim S_{r, t}
$$

The limiting values of the stock price, $S_{r, t}$ and $S_{n, t}$, are given in Equation (13). The limiting values of the instantaneous moments of stock returns are equal to the moments of the corresponding asymptotic expressions above.

Observe that in the first two cases, when the irrational trader is pessimistic or moderately optimistic, the stock price process does not converge quickly to its value in the economy populated exclusively by the trader who survives in the long-run. Instead, over long periods of time, i.e., for $t$ between 0 and $\lambda_{S} T$, the stock price process is affected by the presence of both traders.

We illustrate this property of the stock price in Figure 8 by showing which of the traders has a finite asymptotic impact on the stock price for different combinations of model parameters. We set $\lambda=\frac{2}{3}$ to illustrate that both traders can potentially affect the stock price even if one of them becomes infinitesimally small asymptotically. Eventually, when $t$ reaches $\lambda_{S} T$, convergence of the stock price to its value in a single-trader economy does occur. As we show in the next proposition, in each of the cases considered in Proposition 6, for any non-zero value of lambda, the proportion of aggregate wealth controlled by the trader who does not survive in the long run becomes arbitrarily small by time $t=\lambda T$.

This verifies that the first assumption of the partial equilibrium "invasion" argument is incorrect. As the horizon of the economy expands, a trader can control an asymptotically infinitesimal fraction of the total wealth and yet exert a non-negligible effect on the stock price. In other words, convergence in wealth does not readily imply convergence in prices. 


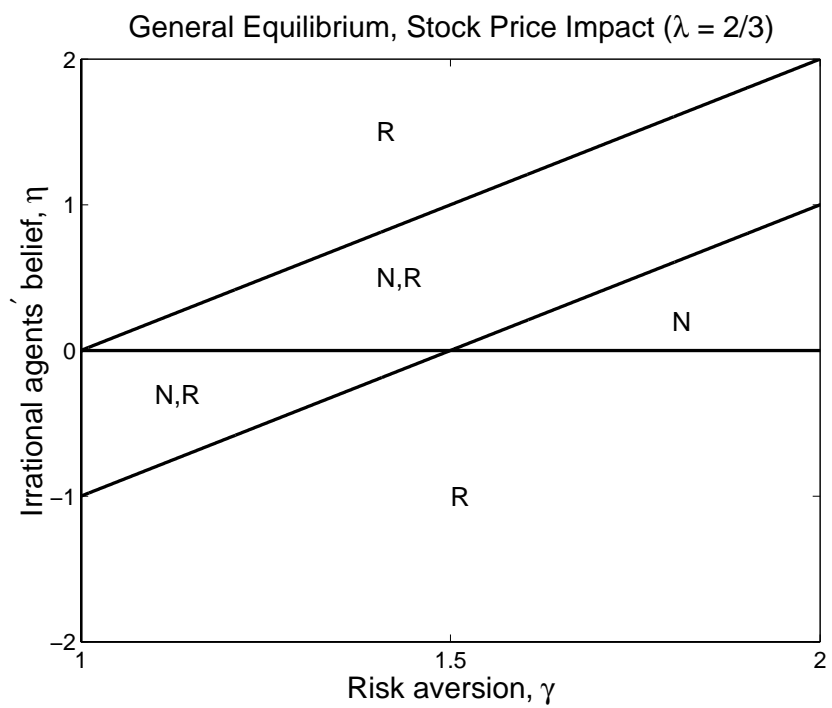

Figure 8: The price impact of rational and irrational traders for different values of $\eta$ and $\gamma$ in general equilibrium. The time is fixed at $t=\frac{2}{3} T$. For each region in the parameter space, we document which of the traders has an impact on the stock price in the long run. "R" means that the rational trader has an impact inside the region, "N" means that the irrational trader has an impact, and "N,R" means that both traders have an impact.

\section{Portfolio Policies}

Proposition 6 in the previous section established the possibility that a trader whose wealth diminishes over time can have a persistent impact on asset prices. In this section, we study in more detail the differences in the survival results between the partial equilibrium and general equilibrium analysis. ${ }^{7}$ In particular, we examine the traders' portfolio policies and show that convergence in the price process does not lead to immediate convergence in policies.

Expressions for portfolio policies are not available in closed form. However, using the same arguments as in the proof of the bounds on the volatility of stock returns in Proposition 1 , it can be shown that individual portfolio holdings are bounded in absolute value, $|w| \leq$ $1+|\eta|(\gamma+1) / \gamma$. The bound on the traders' portfolio holdings is important for our results. It explicitly shows that price impact of the irrational trader with negligible wealth does not rely on excessive leverage. It also implies that our long-run survival results do not rely on the traders' use of high leverage. Our solution for the equilibrium remains valid even if traders are constrained in their portfolio choices, as long as the constraint is sufficiently loose to allow for $w= \pm[1+|\eta|(\gamma+1) / \gamma]$.

\footnotetext{
${ }^{7}$ In a recent paper, Loewenstein and Willard (2002) emphasize the importance of imposing market clearing in all markets for many existing models of mispricing with irrational traders.
} 
The difference in the survival results between the partial equilibrium and general equilibrium analysis occur when $\frac{\gamma}{2 \gamma-1} \eta^{\star}<\eta<\gamma \eta^{\star}$ (see Equations (20) and (21) and Figures 4 and 5). Partial equilibrium argument predicts survival of both traders for these parameter values while general equilibrium analysis shows the extinction of the irrational trader when $\eta>\eta^{\star}$. As mentioned earlier, the partial equilibrium argument relies on assumptions, (i) and (ii), namely, when the irrational trader becomes small in relative wealth, (i) the stock price behaves as if he is absent and (ii) he adopts the portfolio policy that is optimal under that price process. We know that assumption (i) is false in general. But this does not directly explain the discrepancy in survival results. For instance, $\eta^{\star}<\eta<\gamma \eta^{\star}$ corresponds to Case 3 of Proposition 6, in which the stock price is asymptotically the same as in the economy without the irrational trader. In other words, the irrational trader has no significant impact on the current stock price as his wealth becomes negligible. The moments of stock returns converge to the values implied by the partial equilibrium analysis. However, as we show below, the irrational trader's portfolio policy can differ significantly from what the partial equilibrium price process predicts, in contrast to assumption (ii). This explains the different conclusions about traders' survival in the long-run.

To analyze the traders' portfolio policies in more detail, we decompose a trader's stock demand into two components, the myopic component and the hedging component. The sum of the two gives the trader's total stock demand. We have the following proposition.

Proposition 7 (Portfolio Policies) At $=\lambda T$, the individual stock holdings behave as follows when $T \rightarrow \infty$.

Case 1. Pessimistic Irrational Trader $(\eta<0):{ }^{8}$

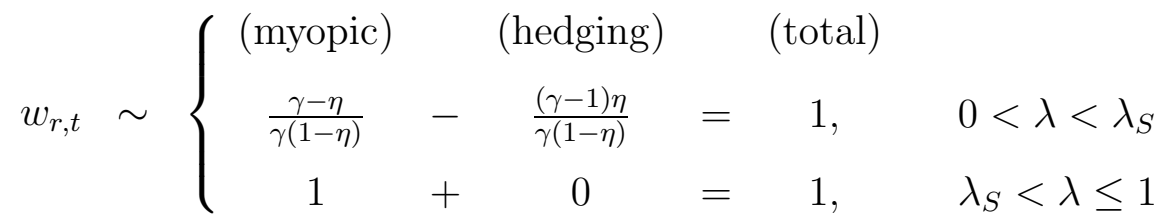

$$
\begin{aligned}
& w_{n, t} \sim\left\{\begin{array}{ccccc}
\text { (myopic) } & & \text { (hedging) } & & \text { (total) } \\
\frac{1}{1-\eta}+ & 0 & \frac{1}{1-\eta}, & & 0<\lambda<\min \left(\lambda_{n}, \lambda_{S}\right) \\
1+\frac{\eta}{\gamma}+ & 0 & 1+\frac{\eta}{\gamma}, & & \max \left(\lambda_{n}, \lambda_{S}\right)<\lambda \leq 1
\end{array}\right.
\end{aligned}
$$

\footnotetext{
${ }^{8}$ The limit of the portfolio policy for values of $\lambda \in\left[\min \left(\lambda_{n}, \lambda_{S}\right), \max \left(\lambda_{n}, \lambda_{S}\right)\right]$ can be characterized explicitly as well, but the results depend on the ordering between $\lambda_{n}$ and $\lambda_{S}$, which in turn is determined by the values of model parameters. We omit these results to simplify the exposition.
} 
Case 2. Moderately Optimistic Irrational Trader $\left(0<\eta<\eta^{\star}\right)$ :

$$
\begin{aligned}
& w_{r, t} \sim\left\{\begin{array}{clrlrl}
\text { (myopic) } & \text { (hedging) } & & \text { (total) } & & \\
\frac{1}{1+\eta}+ & 0 & & \frac{1}{1+\eta}, & & 0<\lambda<\lambda_{r} \\
\frac{1}{1+\eta}+ & \frac{\eta(\gamma-1)}{\gamma(1+\eta)} & & 1-\frac{\eta}{\gamma(1+\eta)}, & & \lambda_{r}<\lambda<\lambda_{S} \\
1-\frac{\eta}{\gamma}+ & 0 & =1-\frac{\eta}{\gamma}, & & \lambda_{S}<\lambda \leq 1
\end{array}\right. \\
& w_{n, t} \sim\left\{\begin{array}{cccc}
\text { (myopic) } & \text { (hedging) } & \text { (total) } & \\
\frac{\gamma+\eta}{\gamma(1+\eta)}+ & =1, & & 0<\lambda<\lambda_{S} \\
1 & +\quad 0 & =1, & \lambda_{S}<\lambda \leq 1
\end{array}\right.
\end{aligned}
$$

Case 3. Strongly Optimistic Irrational Trader, $\left(\eta^{\star}<\eta\right)$ :

$$
\begin{aligned}
& w_{r, t} \sim 1+0=1, \quad 0<\lambda \leq 1 \\
& w_{n, t} \sim\left\{\begin{array}{cccc}
(\text { myopic }) & \text { (hedging) } & \text { (total) } \\
1+\frac{\eta}{\gamma}+ & \frac{\eta(\gamma-1)}{\gamma}=1+\eta, & 0<\lambda<\lambda_{n} \\
1+\frac{\eta}{\gamma}+ & 0 & =1+\frac{\eta}{\gamma}, & \lambda_{n}<\lambda \leq 1
\end{array}\right.
\end{aligned}
$$

Since the moments of stock returns are asymptotically state-independent, it is intuitive to assume, as in assumption (ii), that the implied portfolio policies are myopic. Proposition 7 shows, however, that this is not true. In other words, the asymptotic portfolio policy can differ significantly from what the asymptotic moments of stock returns suggest. The deviation comes from the hedging demand in the traders' portfolio holdings. By definition, the myopic component of portfolio holdings depends only on the instantaneous moments of stock returns. Hence, it converges to its partial equilibrium value as long as the instantaneous moments of stock returns converge. Given that the instantaneous moments of stock returns are asymptotically state-independent, it may seem surprising that the hedging component of portfolio holdings may not converge to zero, as Case 3 of Proposition 7 illustrates for the irrational trader. The reason behind this result is that limiting moments of stock returns do not fully characterize the investment opportunities traders face. In particular, for finite values of $T$, moments of stock returns do not always stay constant. As we have seen in Figure 1, for example, return volatility can change significantly as the relative wealth distribution changes. As $T$ becomes large, the probability for the reversal of wealth distribution between 
the rational and irrational traders and changes in the equilibrium return moments becomes smaller. Nonetheless, the possibility of such a change remains important, which gives rise to the significant hedging demand in the traders' portfolio holdings.

Figure 9 illustrates the behavior of the economy when the irrational trader is strongly optimistic $\left(\eta>\eta^{\star}\right)$. In this case (Case 3 in Propositions 4, 6 and 7), the irrational trader does not survive and has no price impact in the long-run. For the chosen set of parameter values, $\lambda_{n}=0.29$. The time of observation $t$ is set to be $0.15 T$. Thus $t<\lambda_{n} T$. As the bottom panel of Figure 9 shows, with almost probability one, the rational trader controls most of the wealth in the economy by this point in time. As Proposition 6 states, at this point the stock price converge closely to the price in the economy populated by only the rational trader. If we consider the Sharpe ratio of the stock, defined by $\mu_{S} / \sigma_{S}$, which characterizes the instantaneous investment opportunity traders face, it also converges to its value in the limiting economy with the rational trader only, which is $\gamma \sigma$. The top panel plots the value of the Sharpe ratio for different states of the economy at time $t$. It is obvious that with almost probability one, the value of the Sharpe ratio equals the limit $\gamma \sigma$ (the probability distribution of the state of economy is shown by the shaded area). However, for very large values of $D_{t}$ (or $B_{t}$ ), the economy will be dominated by the irrational trader (as we see from the bottom panel) and the instantaneous Sharpe ratio of the stock converges to its value in an economy populated by the irrational trader only, which is $(\gamma-\eta) \sigma$. Such a possibility, even though with very low probability under the true probability measure, can be important to the irrational trade because under his belief, its likelihood can be non-trivial. As a result, it can have a significant impact on the irrational trader's portfolio choice.

The importance of these low probability but large changes in the Sharpe ratio is reflected in the traders' indirect utility function. Since traders in our model have constant relative risk aversion, the indirect utility function of the irrational trader can be expressed in the form

$$
V\left(t, W_{t}, D_{t}\right) \equiv \mathrm{E}_{\mathrm{t}}\left[\xi_{T} \frac{W_{T}^{1-\gamma}}{1-\gamma}\right]=\frac{1}{1-\gamma} e^{h\left(t, D_{t}\right)} W_{t}^{1-\gamma} \equiv \mathrm{E}_{\mathrm{t}}\left[\xi_{T} \frac{1}{1-\gamma} C_{n, T}^{1-\gamma}\right]
$$

State dependence of the indirect utility function, i.e., the effect of possible changes in the Sharpe ratio, is captured by the function $h\left(t, D_{t}\right)$. The second panel of Figure 9 shows that for the irrational trader $h$ is non-constant over a wide range of values of $D_{t}$. It exhibits significant state-dependence even when the contemporaneous Sharpe ratio is approximately constant. It is this state-dependence in the indirect utility function that induces hedging 

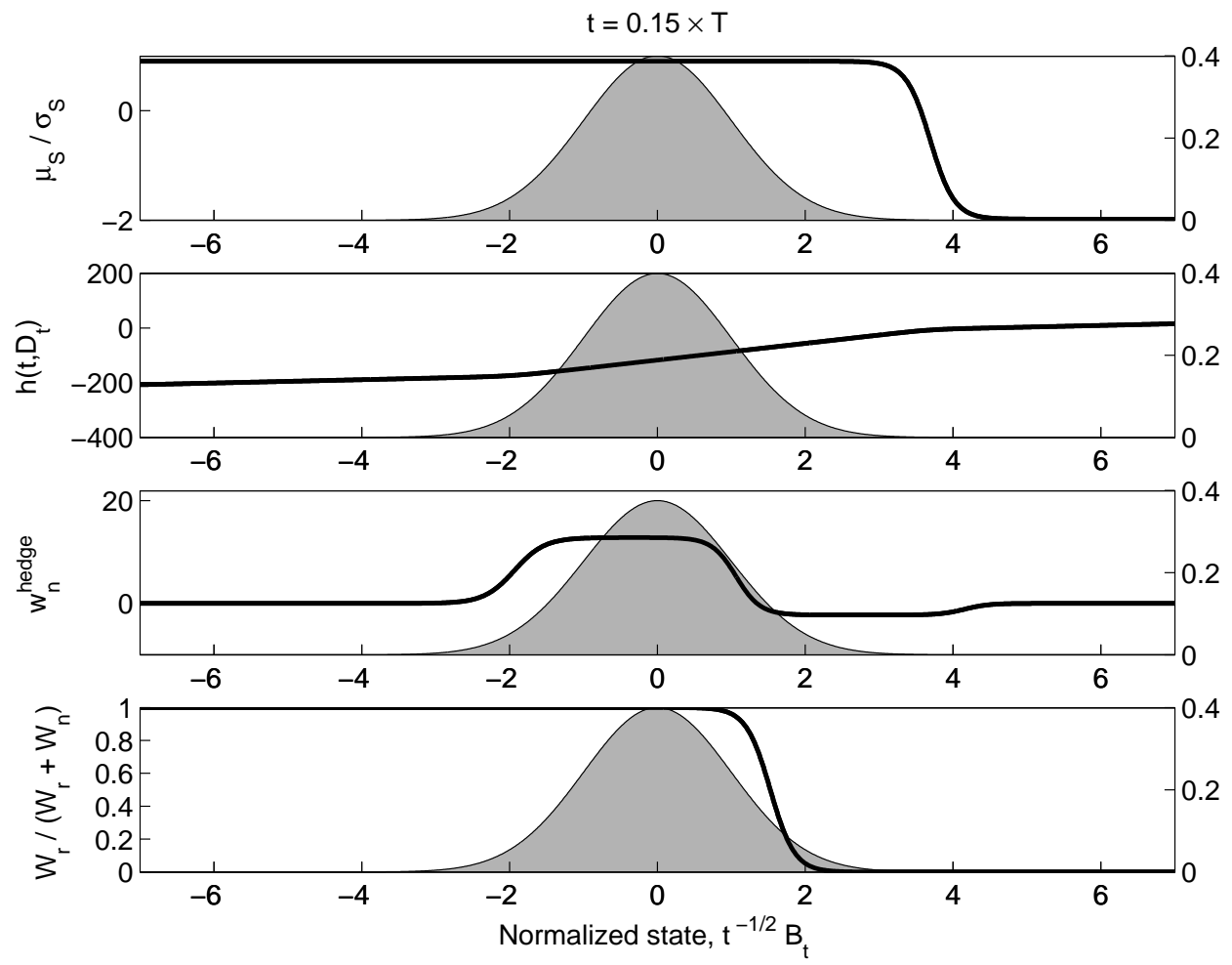

Figure 9: The behavior of the economy for the following parameter values: $\mu=0.12, \sigma=0.18$, $\gamma=5, T=30$. Also, $\eta=2 \eta^{\star}=16$, i.e., the irrational trader is strongly optimistic. The time of observation is set at $t=0.15 \times T$. The horizontal axis in all panels is the normalized state variable, $g_{0, T}=B_{T} / \sqrt{T}$, which has a standard normal distribution with zero mean and unit variance, shown by the shaded area (vertical axis on the right). The four panels from top to bottom show (i) the instantaneous Sharpe ratio of returns, $\mu_{S} / \sigma_{S}$; (ii) the state dependence of the indirect value function of the rational trader, as captured by the function $h\left(t, D_{t}\right)$ in (23); (iii) the portion of the portfolio strategy of the irrational trader attributable to hedging demand, defined as $w_{n}^{\text {hedge }}=$ $w_{n}-\mu_{S}+\eta \sigma_{S}^{2} /\left(\gamma \sigma_{S}^{2}\right)$; (iv) the fraction of the aggregate wealth controlled by the rational agent, $W_{r} /\left(W_{r}+W_{n}\right)$. 
demand. The third panel of Figure 9 shows hedging demand of the irrational trader. Over a wide range of values of $D_{t}$, his hedging demand is non-zero. In particular, it is close to its asymptotic value $\eta(\gamma-1) / \gamma$ (see Proposition 7 ), which equals 12.8 for the chosen values of parameters.

What we conclude from this is that convergence of the stock price to a limiting process does not necessarily imply convergence of the traders' portfolio policies to their policies under the limiting price process. Price paths of small probability under the true probability measure can have a significant impact on the traders' portfolio policies. Thus, assumption (ii) of the partial equilibrium analysis that convergence in price gives convergence in portfolio policies does not hold in general. In the case we are interested in, the irrational trader is strongly optimistic and does not survive in the long-run. As his wealth diminishes, the stock price becomes very close to the price set by the rational trader who dominates the economy and the instantaneous moments of stock returns stay constant with almost probability one. Yet, the irrational trader's portfolio policy remains far from myopic. The presence of the hedging component in his holdings can lower the growth of his portfolio and reduce his chance of recovery from past losses. Such a mechanism is overlooked in the partial equilibrium, which creates the difference in the survival results between partial equilibrium and general equilibrium analysis.

In the appendix, we also examine the dynamics of the wealth distribution of the economy as well as the welfare implications of the irrational beliefs.

\section{Conclusion}

The analysis above has examined the long-run survival of irrational traders who use persistently wrong beliefs to make their portfolio choices. Using a parsimonious model with no intermediate consumption, we have shown that the partial equilibrium arguments to support the long-run survival of irrational traders are flawed and that the impact irrational traders have on equilibrium prices is important in determining their long-run fortunes. In particular, we have found that an irrational trader with a very small wealth may still have a large impact on the stock price, and, furthermore, that irrational traders with beliefs mildly different from the true probabilities can survive in the long run.

For tractability, we confined our analysis to preferences with constant relative risk aversion. Extensions of our analysis to more general preferences are possible and may yield 
unexpected results. We have also assumed that the rational and irrational traders differ only in their beliefs but not in their preferences. This allows us to focus on the impact of irrational beliefs on survival and prices. Of course, differences in time and risk preferences can have their own implications for long-run survival.

Perhaps more important is the extension of these results to models with intermediate consumption and to alternative preferences. We have deferred this to a second paper but, while there is much more to be done on this topic, it is fair to say that a general message is emerging and is unlikely to be overturned. Namely, survival and price impact are related but independent concepts and the arguments of partial equilibrium are not to be trusted. In our model irrational traders could survive and could even dominate rational traders, but, contrary to the partial equilibrium analysis, even if they did not survive, they could still have a persistent impact on prices. 


\section{A Wealth Distribution and Welfare}

\section{Wealth Distribution}

Section 5 discussed the limiting distribution of wealth between the rational and irrational traders at the final date $T$, as $T$ approached infinity. We now characterize the dynamics of the wealth distribution in the economy.

Proposition 8 (Individual Wealth) At $t=\lambda T$, the individual wealth processes behave as follows when $T \rightarrow \infty$.

Case 1. Pessimistic Irrational Trader $(\eta<0)$ :

$$
\begin{aligned}
& \frac{W_{r, t}}{W_{n, t}} \sim \begin{cases}e^{\frac{1}{2}[\eta-2(\gamma-1)] \eta \sigma^{2} t-\eta \sigma B_{t}}, & 0<\lambda<\lambda_{n} \\
e^{\frac{1}{2}\left(\eta^{2} / \gamma^{2}\right)(\gamma-1) \sigma^{2} T+\frac{1}{2}\left[\left(\eta^{2} / \gamma^{2}\right)-2 \eta(\gamma-1) / \gamma\right] \sigma^{2} t-(\eta / \gamma) \sigma B_{t}}, & \lambda_{n}<\lambda \leq 1\end{cases} \\
& W_{r, t} \sim S_{t}
\end{aligned}
$$

Case 2. Moderately Optimistic Irrational Trader $\left(0<\eta<\eta^{\star}\right)$ :

$$
\begin{aligned}
& \frac{W_{r, t}}{W_{n, t}} \sim \begin{cases}e^{\frac{1}{2}[\eta-2(\gamma-1)] \eta \sigma^{2} t-\eta \sigma B_{t}}, & 0<\lambda<\lambda_{r} \\
e^{-\frac{1}{2}\left(\eta^{2} / \gamma^{2}\right)(\gamma-1) \sigma^{2} T+\frac{1}{2}\left[\left(\eta^{2} / \gamma^{2}\right)(2 \gamma-1)-2 \eta(\gamma-1) / \gamma\right] \sigma^{2} t-(\eta / \gamma) \sigma B_{t}}, & \lambda_{r}<\lambda \leq 1\end{cases} \\
& W_{n, t} \sim S_{t}
\end{aligned}
$$

Case 3. Strongly Optimistic Irrational Trader $\left(\eta^{\star}<\eta\right)$ :

$$
\begin{aligned}
& \frac{W_{r, t}}{W_{n, t}} \sim \begin{cases}e^{\frac{1}{2}[\eta-2(\gamma-1)] \eta \sigma^{2} t-\eta \sigma B_{t}}, & 0<\lambda<\lambda_{n} \\
e^{\frac{1}{2}\left(\eta^{2} / \gamma^{2}\right)(\gamma-1) \sigma^{2} T+\frac{1}{2}\left[\left(\eta^{2} / \gamma^{2}\right)-2 \eta(\gamma-1) / \gamma\right] \sigma^{2} t-(\eta / \gamma) \sigma B_{t}}, & \lambda_{n}<\lambda \leq 1\end{cases} \\
& W_{r, t} \sim S_{t}
\end{aligned}
$$

The results for Case 3 confirms our analysis of the difference in the irrational trader's survival between general and partial equilibrium analysis. Despite the convergence of the stock price to its limiting process, the irrational trader does not follow a myopic portfolio policy as the limiting price process implies. The hedging demand in his portfolio policy 
changes the growth rate of his wealth from that of the myopic policy and the chance of his survival.

Lastly, we show that even when the stock price, the traders' portfolio policies and their wealth eventually do converge to their limiting values, the irrational trader suffered from wealth losses may not recover, in contrast to the partial equilibrium argument. Consider a combination of model parameters satisfying $\eta^{\star}<\eta<\gamma \eta^{\star}$. In this case, for sufficiently large values of $t$, the stock price, the traders' portfolio policies, and the growth rate of their wealth do eventually converge to their partial equilibrium values as Propositions 6 and 7 show. Yet, from Proposition 8, the irrational trader still becomes extinct in the long-run. The reason for this can be seen from Proposition 8 and is further illustrated in Figure 10 (the second curve from the top). Even though for $t=\lambda T, \lambda>\lambda_{n}$, the relative wealth of the irrational trader is growing, it does so only following a period of decline, i.e., $0<\lambda<\lambda_{n}$. This happens because assumption (ii) of the partial equilibrium analysis does not hold for $0<\lambda<\lambda_{n}$ and the irrational trader suffers significant decline in relative wealth growth this period. If this decline is below certain threshold, he will be unable to recover despite relative growth afterwards and cannot escape from relative extinction.

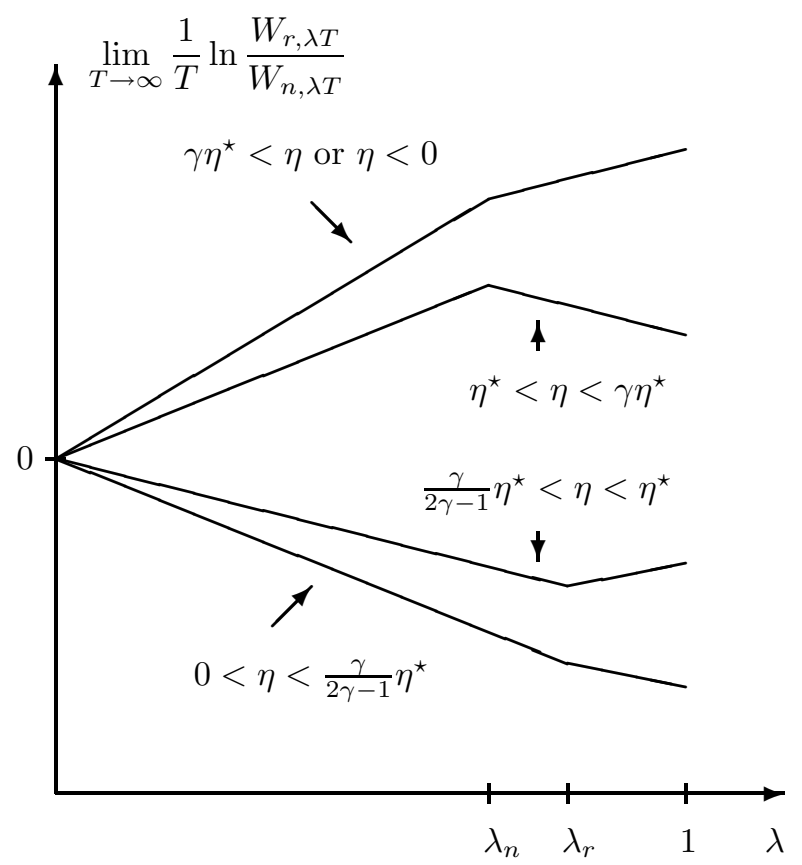

Figure 10: Asymptotic Wealth Behavior. 


\section{Utility Loss of Irrational Beliefs}

As we have established, the irrational trader can have a significant impact on the behavior of stock returns. In this subsection we show that the impact on prices is associated with utility loss by irrational traders. By revealed preference, the rational trader always benefits from the presence of irrational traders, since the autarchic solution remains feasible in our equilibrium economy. We quantify this benefit and show that it is asymptotically independent of the exact belief of the irrational traders, and, surprisingly, therefore independent of the long run survival of the rational trader.

For $\gamma>1$ and $\eta \neq 0$, the certainty equivalent of the terminal consumption of the rational trader in the equilibrium economy we are analyzing is given by

$$
\text { C.E. }\left(C_{r, T}\right) \equiv\left(\mathrm{E}_{0}\left[C_{r, T}^{1-\gamma}\right]\right)^{\frac{1}{1-\gamma}} \sim e^{\left(\mu-\frac{1}{2} \sigma^{2} \gamma\right) T} .
$$

The above expression is the same as the certainty equivalent of consuming the entire aggregate dividend at time T. Thus, we find that, in the limit of economy horizon approaching infinity, the rational trader realizes the largest possible benefit of risk sharing, and this conclusion is independent of the exact belief bias $\eta$ exhibited by the irrational trader. This remains the case even if the rational trader does not survive in the long run.

Next, we compute the certainty equivalent of the consumption of the irrational trader. We are using the objective probability distribution in our calculation, i.e., we are finding the certainty equivalent the the rational trader would assign to the terminal consumption profile of the irrational trader. As before, we assume $\gamma>1$ and $\eta \neq 0$. We find

$$
\text { C.E. }\left(C_{n, T}\right) \equiv\left(\mathrm{E}_{0}\left[C_{n, T}^{1-\gamma}\right]\right)^{\frac{1}{1-\gamma}} \sim e^{\left(\mu-\frac{1}{2} \sigma^{2} \gamma-\frac{1}{2} \eta^{2} \sigma^{2}(2 \gamma-1) / \gamma^{2}\right) T} .
$$

We know from our previous analysis that the irrational traders can have long-run impact on the asset prices in equilibrium. To quantify this impact, we consider that certainty equivalent of the consumption chosen by the irrational trader facing the prices free of their impact on prices, i.e.., the prices in the economy populated exclusively by rational traders. Assuming that the endowment of the irrational trader is one half of the aggregate dividend, a straightforward calculation yields the certainty equivalent of

$$
\frac{1}{2} e^{\left(\mu-\frac{1}{2} \sigma^{2} \gamma-\frac{1}{2} \eta^{2} \sigma^{2} \frac{1}{\gamma}\right) T}
$$

Since $\gamma>1$, the above expression has higher growth rate in $T$ than (24), i.e., asymptotically 
the irrational traders are hurt by their own price impact. As a quantitative measure of the resulting utility loss one can use the fraction of the aggregate dividend with which the irrational agent must be endowed in an economy without price impact to achieve the same certainty equivalent consumption as in our general equilibrium economy. We find that this fraction converges to zero asymptotically.

\section{B Proofs}

\section{Proof of Proposition 1}

The optimality conditions of the maximization problem in (9a) require that

$$
C_{r, T}=C_{n, T}\left(b \xi_{T}\right)^{1 / \gamma}
$$

Combined with the market clearing condition (9b), this implies (10a) and (10b).

The state price density must be proportional to the traders' marginal utilities. Since we set the interest rate equal to zero, the state price density conditional on the information available at time $t$ is given by

$$
\frac{\left(1+\left(b \xi_{T}\right)^{1 / \gamma}\right)^{\gamma} D_{T}^{-\gamma}}{\mathrm{E}_{\mathrm{t}}\left[\left(1+\left(b \xi_{T}\right)^{1 / \gamma}\right)^{\gamma} D_{T}^{-\gamma}\right]}
$$

The price of any payoff $Z_{T}$ is therefore given by (12).

The individual budget constraint in a dynamically complete market is equivalent to the static constraint that the initial wealth of an trader is equal to the present value of the trader's consumption (e.g., Cox and Huang (1989). Since the two traders in our model have identical endowments at time $t=0$, their budget constraints imply

$$
W_{r, 0}=\frac{\mathrm{E}_{0}\left[D_{T}^{1-\gamma}\left(1+\left(b \xi_{T}\right)^{\frac{1}{\gamma}}\right)^{\gamma-1}\right]}{\mathrm{E}_{0}\left[D_{T}^{-\gamma}\left(1+\left(b \xi_{T}\right)^{\frac{1}{\gamma}}\right)^{\gamma}\right]}=\frac{\mathrm{E}_{0}\left[D_{T}^{1-\gamma}\left(b \xi_{T}\right)^{\frac{1}{\gamma}}\left(1+\left(b \xi_{T}\right)^{\frac{1}{\gamma}}\right)^{\gamma-1}\right]}{\mathrm{E}_{0}\left[D_{T}^{-\gamma}\left(1+\left(b \xi_{T}\right)^{\frac{1}{\gamma}}\right)^{\gamma}\right]}=W_{n, 0}
$$

We now verify that $b=e^{\eta \sigma^{2}(\gamma-1) T}$ satisfies $(25)$. Note that

$$
D_{T}^{1-\gamma}=e^{\left[(1-\gamma)\left(\mu-\frac{\sigma^{2}}{2}\right)+\frac{1}{2}(1-\gamma)^{2} \sigma^{2}\right] T} e^{-\frac{1}{2}(1-\gamma)^{2} \sigma^{2} T+(1-\gamma) \sigma B_{T}}
$$


where the second term is an exponential martingale. Define a new measure $Q$, such that

$$
\left(\frac{d Q}{d P}\right)_{t}=e^{-\frac{1}{2}(1-\gamma)^{2} \sigma^{2} t+(1-\gamma) \sigma B_{t}}
$$

where $P$ is the original probability measure. By Girsanov's theorem, the process

$$
B_{t}^{Q}=B_{t}-(1-\gamma) \sigma t
$$

is a Brownian motion under Q. Thus, Girsanov's theorem implies that the equality

$$
\mathrm{E}_{0}\left[D_{T}^{1-\gamma}\left(b \xi_{T}\right)^{\frac{1}{\gamma}}\left(1+\left(b \xi_{T}\right)^{\frac{1}{\gamma}}\right)^{\gamma-1}\right]=\mathrm{E}_{0}\left[D_{T}^{1-\gamma}\left(1+\left(b \xi_{T}\right)^{\frac{1}{\gamma}}\right)^{\gamma-1}\right]
$$

is equivalent to

$$
\mathrm{E}_{0}^{Q}\left[\left(\xi_{T}^{Q}\right)^{\frac{1}{\gamma}}\left(1+\left(\xi_{T}^{Q}\right)^{\frac{1}{\gamma}}\right)^{\gamma-1}\right]=\mathrm{E}_{0}^{Q}\left[\left(1+\left(\xi_{T}^{Q}\right)^{\frac{1}{\gamma}}\right)^{\gamma-1}\right]
$$

where $\xi_{t}^{Q}=\exp \left(-\frac{1}{2} \sigma^{2} \eta^{2} t+\sigma \eta B_{t}^{Q}\right)$. Since the process $B_{t}^{Q}$ is equivalent in distribution to $B_{t}$, we can restate the last equality equivalently as

$$
\mathrm{E}_{0}\left[\xi_{T}^{\frac{1}{\gamma}}\left(1+\xi_{T}^{\frac{1}{\gamma}}\right)^{\gamma-1}\right]=\mathrm{E}_{0}\left[\left(1+\xi_{T}^{\frac{1}{\gamma}}\right)^{\gamma-1}\right]
$$

To verify that the above equality holds, consider a function $F(z)$ defined as

$$
F(z)=\mathrm{E}_{0}\left[\left(e^{\frac{1}{2 \gamma} z T}+e^{-\frac{1}{2 \gamma} z T} \xi_{T}^{\frac{1}{\gamma}}\right)^{\gamma}\right]
$$

Changing the order of differentiation and expectation operators, (see Billingsley 1995, Th. 16.8),

$$
\left.F^{\prime}(z)\right|_{z=0}=\mathrm{E}\left[\frac{1}{2}\left(1-\xi_{T}^{\frac{1}{\gamma}}\right)\left(1+\xi_{T}^{\frac{1}{\gamma}}\right)^{\gamma-1}\right]
$$

Thus it suffices to prove that $\left.F^{\prime}(z)\right|_{z=0}=0$.

Since

$$
\mathrm{E}_{0}\left[\left(e^{\frac{1}{2 \gamma} z T}+e^{-\frac{1}{2 \gamma} z T} \xi_{T}^{\frac{1}{\gamma}}\right)^{\gamma}\right]=\mathrm{E}_{0}\left[\left(e^{\frac{1}{2 \gamma}\left(z T-\frac{1}{2} \eta^{2} \sigma^{2} T+\eta \sigma B_{T}\right)}+e^{-\frac{1}{2 \gamma}\left(z T-\frac{1}{2} \eta^{2} \sigma^{2} T+\eta \sigma B_{T}\right)}\right)^{\gamma} \xi_{T}^{\frac{1}{2}}\right]
$$


if we define a new measure $Q$ so that

$$
\left(\frac{d Q}{d P}\right)_{t}=e^{-\frac{1}{8} \eta^{2} \sigma^{2} t+\frac{1}{2} \eta \sigma B_{t}}
$$

and use Girsanov's theorem in a manner similar to its earlier application in this proof, we find that (27) equals

$$
\mathrm{E}_{0}\left[\left(e^{\frac{1}{2 \gamma}\left(z T+\eta \sigma B_{T}\right)}+e^{-\frac{1}{2 \gamma}\left(z T+\eta \sigma B_{T}\right)}\right)^{\gamma}\right] e^{-\frac{1}{8} \eta^{2} \sigma^{2} T} .
$$

The symmetry of the distribution of the normal random variable $B_{T}$ implies that $F(z)=$ $F(-z)$, therefore $\left.F^{\prime}(z)\right|_{z=0}=0$. This verifies that $b=e^{\eta \sigma^{2}(\gamma-1) T}$.

The stock price is given by

$$
S_{t}=\frac{\mathrm{E}_{\mathrm{t}}\left[D_{T}^{1-\gamma}\left(1+\left(b \xi_{T}\right)^{1 / \gamma}\right)^{\gamma}\right]}{\mathrm{E}_{\mathrm{t}}\left[D_{T}^{-\gamma}\left(1+\left(b \xi_{T}\right)^{1 / \gamma}\right)^{\gamma}\right]}
$$

Define

$$
\begin{aligned}
& A=e^{\left(\frac{-\eta \sigma^{2}}{\gamma}\right)(T-t)} \\
& g=e^{-\frac{1}{2} \eta^{2} \sigma^{2} \frac{1}{\gamma} T+\sigma^{2} \eta(\gamma-1) \frac{1}{\gamma} t+\frac{\eta \sigma}{\gamma} B_{T}}
\end{aligned}
$$

then, as we have shown above, the stock price can be equivalently expressed as

$$
S_{t}=e^{\left(\mu-\sigma^{2} \gamma\right) T+\left(-\frac{1}{2} \sigma^{2}(1-2 \gamma)\right) t} e^{\sigma B_{t}} \frac{\mathrm{E}_{\mathrm{t}}\left[(1+g)^{\gamma}\right]}{\mathrm{E}_{\mathrm{t}}\left[(1+g A)^{\gamma}\right]}
$$

and therefore, by Ito's lemma, its volatility $\sigma_{S t}$ is given by

$$
\sigma_{S t}=\frac{\partial \ln S_{t}}{\partial B_{t}}=\sigma+\eta \sigma\left(\frac{\mathrm{E}_{\mathrm{t}}\left[(1+g A)^{\gamma-1}\right]}{\mathrm{E}_{\mathrm{t}}\left[(1+g A)^{\gamma}\right]}-\frac{\mathrm{E}_{\mathrm{t}}\left[(1+g)^{\gamma-1}\right]}{\mathrm{E}_{\mathrm{t}}\left[(1+g)^{\gamma}\right]}\right) .
$$

To establish the bounds on volatility, we prove that

$$
\frac{\mathrm{E}_{\mathrm{t}}\left[(1+g A)^{\gamma-1}\right]}{\mathrm{E}_{\mathrm{t}}\left[(1+g A)^{\gamma}\right]}-\frac{\mathrm{E}_{\mathrm{t}}\left[(1+g)^{\gamma-1}\right]}{\mathrm{E}_{\mathrm{t}}\left[(1+g)^{\gamma}\right]} \geq 0
$$

for $A \leq 1$ with the opposite inequality for $A \geq 1$. Note that for any twice-differentiable 
function $F(A, \gamma)$,

$\frac{\partial}{\partial \gamma} \frac{\partial}{\partial A} \ln (F(A, \gamma)) \geq 0 \Rightarrow \frac{\partial}{\partial A} \ln (F(A, \gamma-1))-\frac{\partial}{\partial A} \ln (F(A, \gamma)) \leq 0 \Rightarrow \frac{\partial}{\partial A} \frac{F(A, \gamma-1)}{F(A, \gamma)} \leq 0$

Thus, to prove (29), it suffices to show that $\partial^{2} \ln \left(\mathrm{E}_{\mathrm{t}}\left[(1+g A)^{\gamma}\right]\right) / \partial A \partial \gamma \geq 0$. The function $(1+g A)^{\gamma}$ is log-supermodular in $A, g$, and $\gamma$, since it is positive and it's cross-partial derivatives in all arguments are positive. Thus, according to the additivity property of logsupermodular functions (see Athey (2002)), $\mathrm{E}_{\mathrm{t}}\left[(1+g A)^{\gamma}\right]$ is log-supermodular in $A$ and $\gamma$, i.e., $\partial^{2} \ln \left(\mathrm{E}_{\mathrm{t}}\left[(1+g A)^{\gamma}\right]\right) / \partial A \partial \gamma \geq 0$.

Because $A>1$ if and only if $\eta<0$, we have shown that

$$
\eta\left(\frac{\mathrm{E}_{\mathrm{t}}\left[(1+g A)^{\gamma-1}\right]}{\mathrm{E}_{\mathrm{t}}\left[(1+g A)^{\gamma}\right]}-\frac{\mathrm{E}_{\mathrm{t}}\left[(1+g)^{\gamma-1}\right]}{\mathrm{E}_{\mathrm{t}}\left[(1+g)^{\gamma}\right]}\right) \geq 0
$$

and hence $\sigma_{S t} \geq \sigma$.

Because $\left(\frac{\mathrm{E}_{\mathrm{t}}\left[(1+g A)^{\gamma-1}\right]}{\mathrm{E}_{\mathrm{t}}\left[(1+g A)^{\gamma}\right]}-\frac{\mathrm{E}_{\mathrm{t}}\left[(1+g)^{\gamma-1}\right]}{\mathrm{E}_{\mathrm{t}}\left[(1+g)^{\gamma}\right]}\right)$ is bounded between -1 and 0 for $\eta<0$ and between 0 and 1 for $\eta>0$, we obtain the stated upper bound from (28): $\sigma_{S t} \leq \sigma(1+|\eta|)$.

\section{Proof of Proposition 4}

According to (10a) and (10b),

$$
\frac{C_{n, T}}{C_{r, T}}=\left(b \xi_{T}\right)^{1 / \gamma}=\exp \left[\frac{1}{\gamma}\left(-\frac{1}{2} \sigma^{2} \eta^{2}+\eta \sigma^{2}(\gamma-1)\right) T+\frac{1}{\gamma} \eta \sigma B_{T}\right] .
$$

Using the strong Law of Large Numbers for Brownian motion (see Karatzas and Shreve (1991, Sec. 2.9.A)), for any value of $\sigma$,

$$
\lim _{T \rightarrow \infty} e^{a T+\sigma B_{T}}= \begin{cases}0, & a<0 \\ \infty, & a>0\end{cases}
$$

where convergence takes place almost surely. The statement of the proposition then follows. 


\section{Proof of Proposition 5}

Equations (10a) and (10b) state that

$$
\begin{aligned}
C_{r, T} & =\frac{1}{1+\left(b \xi_{T}\right)^{1 / \gamma}} D_{T} \\
C_{n, T} & =\frac{\left(b \xi_{T}\right)^{1 / \gamma}}{1+\left(b \xi_{T}\right)^{1 / \gamma}} D_{T}
\end{aligned}
$$

If $\mu>\frac{1}{2} \sigma^{2}$, then the irrational trader's consumption converges to zero if and only if $\left(b \xi_{T}\right)^{1 / \gamma} D_{T}$ converges to zero:

$$
\left(b \xi_{T}\right)^{1 / \gamma} D_{T}=\exp \left[\left(\eta \sigma^{2} \frac{1}{\gamma}(\gamma-1)-\frac{1}{2} \eta^{2} \sigma^{2} \frac{1}{\gamma}+\mu-\frac{1}{2} \sigma^{2}\right) T+\left(\sigma \eta \frac{1}{\gamma}+\sigma\right) B_{T}\right] .
$$

Similarly, the rational trader's consumption converges to zero if and only if $\frac{D_{T}}{\left(b \xi_{T}\right)^{1 / \gamma}}$ converges to zero:

$$
\frac{D_{T}}{\left(b \xi_{T}\right)^{1 / \gamma}}=\exp \left[\left(-\eta \sigma^{2} \frac{1}{\gamma}(\gamma-1)+\frac{1}{2} \eta^{2} \sigma^{2} \frac{1}{\gamma}+\mu-\frac{1}{2} \sigma^{2}\right) T+\left(-\sigma \eta \frac{1}{\gamma}+\sigma\right) B_{T}\right] .
$$

According to (30), (31) and (32) imply that

$$
\begin{gathered}
C_{n, T} \rightarrow 0 \Leftrightarrow \gamma\left(2 \frac{\mu}{\sigma^{2}}-1\right)<-2 \eta(\gamma-1)+\eta^{2} \\
C_{r, T} \rightarrow 0 \Leftrightarrow \gamma\left(2 \frac{\mu}{\sigma^{2}}-1\right)<2 \eta(\gamma-1)-\eta^{2}
\end{gathered}
$$

The right hand side of (33a) is convex in $\eta$, thus $C_{n, T} \rightarrow 0$ for $\eta<(\gamma-1)-\sqrt{(\gamma-1)^{2}+\gamma\left(2 \frac{\mu}{\sigma^{2}}-1\right)}$ and $\eta>(\gamma-1)+\sqrt{(\gamma-1)^{2}+\gamma\left(2 \frac{\mu}{\sigma^{2}}-1\right)}$.

Similarly, $C_{n, T} \rightarrow 0$ for $\eta>(\gamma-1)-\sqrt{(\gamma-1)^{2}-\left(2 \frac{\mu}{\sigma^{2}}-1\right)}$ and $\eta<(\gamma-1)+$ $\sqrt{(\gamma-1)^{2}-\gamma\left(2 \frac{\mu}{\sigma^{2}}-1\right)}$. Such a value of $\eta$ exists as long as $(\gamma-1)^{2}-\gamma\left(2 \frac{\mu}{\sigma^{2}}-1\right)>0$.

\section{Proof of Propositions 6, 8}

Our analysis will make use of the following technical result.

Lemma 1 Consider a stochastic process $X_{t}=e^{c t+v B_{t}}$ and a constant $a \geq 0$. Assume that $a c+\frac{1}{2} v^{2} a^{2}(1-\lambda) \neq 0,0 \leq \lambda<1$. Then the limit $\lim _{T \rightarrow \infty} \mathrm{E}_{t}\left[X_{T}^{a}\right]$ is equal to either zero or infinity almost surely, where we set $t=\lambda T$. The following convergence results hold: 
(i) (Point-wise convergence)

$$
\lim _{T \rightarrow \infty} \frac{\mathrm{E}_{t}\left[\left(1+X_{T}\right)^{a}\right]}{1+\mathrm{E}_{t}\left[X_{T}^{a}\right]}=1 .
$$

(ii) (Convergence of moments)

$$
\begin{aligned}
& \lim _{T \rightarrow \infty} \frac{\operatorname{mean}_{\mathrm{t}} \mathrm{E}_{t}\left[\left(1+X_{T}\right)^{a}\right]}{\operatorname{mean}_{\mathrm{t}}\left(1+\mathrm{E}_{t}\left[X_{T}^{a}\right]\right)}=1 \\
& \lim _{T \rightarrow \infty} \frac{\operatorname{vol}_{\mathrm{t}} \mathrm{E}_{t}\left[\left(1+X_{T}\right)^{a}\right]}{\operatorname{vol}_{\mathrm{t}}\left(1+\mathrm{E}_{t}\left[X_{T}^{a}\right]\right)}=1
\end{aligned}
$$

where mean $_{\mathrm{t}} \mathrm{f}_{\mathrm{t}}$ and $\mathrm{vol}_{\mathrm{t}} \mathrm{f}_{\mathrm{t}}$ denote the instantaneous mean and standard deviation of the process $\ln f_{t}$ respectively.

\section{Proof of Lemma 1}

(i) Consider the conditional expectation

$$
\mathrm{E}_{\mathrm{t}}\left[X_{T}^{a}\right]=\exp \left[a c T+\frac{1}{2} v^{2} a^{2}(1-\lambda) T+a v B_{t}\right] .
$$

The limit of $\mathrm{E}_{t}\left[X_{T}^{a}\right]$ is equal to zero if $a c+\frac{1}{2} v^{2} a^{2}(1-\lambda)<0$ and equal to infinity if the opposite inequality holds (according to the strong Law of Large Numbers for Brownian motion, see Karatzas and Shreve, 1991, Sec. 2.9.A).

Because the function $a c T+\frac{1}{2} v^{2} a^{2}(1-\lambda) T$ is convex in $a$ and equal to zero when $a=0$, we find that for $a \geq 1$

$$
\begin{aligned}
& \mathrm{E}_{t}\left[X_{T}^{a}\right] \rightarrow \infty \Rightarrow \frac{\mathrm{E}_{t}\left[X_{T}^{z}\right]}{\mathrm{E}_{t}\left[X_{T}^{a}\right]} \rightarrow 0, \quad \forall z \in(0, a) \\
& \mathrm{E}_{t}\left[X_{T}^{a}\right] \rightarrow 0 \Rightarrow \mathrm{E}_{t}\left[X_{T}^{z}\right] \rightarrow 0, \quad \forall z \in(0, a) .
\end{aligned}
$$

We prove the result of the lemma separately for six regions that cover the entire parameter space.

Case 1: $0 \leq a \leq 1, \mathrm{E}_{t}\left[X_{T}^{a}\right] \rightarrow \infty$

If $X_{T} \leq 1,\left(X_{T}+1\right)^{a} \leq 2^{a}$, while if $X_{T} \geq 1 \Rightarrow\left(X_{T}+1\right)^{a}-X_{T}^{a} \leq a X_{T}^{a-1} \leq a$ since $\left(X_{T}+1\right)^{a}$ is concave and $a-1 \leq 0$. Therefore, $X_{T}^{a} \leq\left(1+X_{T}\right)^{a} \leq X_{T}^{a}+2^{a}+a$, and hence $\lim _{T \rightarrow \infty} \mathrm{E}_{t}\left[\left(1+X_{T}\right)^{a}\right] / \mathrm{E}_{t}\left[X_{T}^{a}\right]=1$, which implies $\lim _{T \rightarrow \infty} \mathrm{E}_{t}\left[\left(1+X_{T}\right)^{a}\right] /\left(1+\mathrm{E}_{t}\left[X_{T}^{a}\right]\right)=1$. 
Case 2: $1 \leq a \leq 2, \mathrm{E}_{t}\left[X_{T}^{a}\right] \rightarrow \infty$

By the mean value theorem, $\left(1+X_{T}\right)^{a}=X_{T}^{a}+a\left(w+X_{T}\right)^{a-1}$ for some $w \in[0,1]$. Using the analysis of case $1,\left(w+X_{T}\right)^{a-1} \leq\left(1+X_{T}\right)^{a-1} \leq X_{T}^{a-1}+2^{a-1}+a-1$, which, combined with (38), implies that $\lim _{T \rightarrow \infty} \mathrm{E}_{\mathrm{t}}\left[\left(1+X_{T}\right)^{a}\right] / \mathrm{E}_{\mathrm{t}}\left[X_{T}^{a}\right]=1$ and the main result follows.

Case 3: $2 \leq a, \mathrm{E}_{\mathrm{t}}\left[X_{T}^{a}\right] \rightarrow \infty$.

By the mean value theorem, $\left(1+X_{T}\right)^{a}=X_{T}^{a}+a\left(w+X_{T}\right)^{a-1}$ for some $w \in[0,1]$. By Jensen's inequality, $\left(\left(1+X_{T}\right) / 2\right)^{a-1} \leq\left(1+X_{T}^{a-1}\right) / 2$. Thus,

$$
0 \leq\left(w+X_{T}\right)^{a-1} \leq\left(1+X_{T}\right)^{a-1} \leq 2^{a-2}+2^{a-2} X_{T}^{a-1}
$$

which, combined with (38) implies that $\lim _{T \rightarrow \infty} \mathrm{E}_{t}\left[\left(1+X_{T}\right)^{a}\right] / \mathrm{E}_{t}\left[X_{T}^{a}\right]=1$ and the main result follows.

Case 4: $0 \leq a \leq 1, \mathrm{E}_{\mathrm{t}}\left[X_{T}^{a}\right] \rightarrow 0:$

If $X_{T} \leq 1,\left(1+X_{T}\right)^{a} \leq 1+X_{T} \leq 1+X_{T}^{a}$, while if $X_{T} \geq 1,\left(1+X_{T}\right)^{a} \leq X_{T}^{a}+$ $a \leq 1+X_{T}^{a}$ since $\left(1+X_{T}\right)^{a}$ is concave. Thus, $1 \leq\left(1+X_{T}\right)^{a} \leq 1+X_{T}^{a}$ and therefore $\lim _{T \rightarrow \infty} \mathrm{E}_{\mathrm{t}}\left[\left(1+X_{T}\right)^{a}\right]=1$, which implies the main result.

Case 5: $1 \leq a \leq 2, \mathrm{E}_{\mathrm{t}}\left[X_{T}^{a}\right] \rightarrow 0$

By the mean value theorem, $\left(1+X_{T}\right)^{a}=1+a X_{T}\left(1+w X_{T}\right)^{a-1}$ for some $w \in[0,1]$. Further, $X_{T}\left(1+w X_{T}\right)^{a-1} \leq X_{T}\left(1+X_{T}\right)^{a-1} \leq X_{T}\left(X_{T}^{a-1}+2^{a-1}+a-1\right)$, using the same argument as in case 1 . Since $\lim _{T \rightarrow \infty} \mathrm{E}_{t}\left[X_{T}^{a}\right]=0$, according to $(39), \lim _{T \rightarrow \infty} \mathrm{E}_{t}\left[X_{T}\right]=0$ and hence $\lim _{T \rightarrow \infty} \mathrm{E}_{\mathrm{t}}\left[\left(1+X_{T}\right)^{a}\right]=1$.

Case 6: $2 \leq a, \mathrm{E}_{\mathrm{t}}\left[X_{T}^{a}\right] \rightarrow 0$.

By the mean value theorem, $\left(1+X_{T}\right)^{a}=1+a X_{T}\left(1+w X_{T}\right)^{a-1}$ for some $w \in[0,1]$. Further, $X_{T}\left(1+w X_{T}\right)^{a-1} \leq X_{T}\left(1+X_{T}\right)^{a-1} \leq 2^{a-2} X_{T}+2^{a-2} X_{T}^{a}$ by Jensen's inequality. Since $\lim _{T \rightarrow \infty} \mathrm{E}_{t}\left[X_{T}^{a}\right]=0$, according to (39), and $\lim _{T \rightarrow \infty} \mathrm{E}_{t}\left[X_{T}\right]=0$ and hence $\lim _{T \rightarrow \infty} \mathrm{E}_{\mathrm{t}}\left[\left(1+X_{T}\right)^{a}\right]=1$.

(ii) Since the conditional expectations $\mathrm{E}_{t}\left[\left(1+X_{T}\right)^{a}\right]$ and $\mathrm{E}_{t}\left[1+X_{T}^{a}\right]$ are martingales, they have zero drift for all values of $T$ and $t$. By Ito's lemma, convergence of the first moments of the natural logarithms of the same processes follows from convergence of the second moments.

We now establish convergence of volatility of the process $\mathrm{E}_{t}\left[\left(1+X_{T}\right)^{a}\right]$. According to 
Ito's lemma, one must show that

$$
\lim _{T \rightarrow \infty} \frac{\partial \ln \mathrm{E}_{t}\left[\left(1+X_{T}\right)^{a}\right] / \partial B_{t}}{\partial \ln \left(1+\mathrm{E}_{t}\left[X_{T}^{a}\right]\right) / \partial B_{t}}=1, \quad \forall a \geq 0 .
$$

Given (37), it suffices to prove that $\lim _{T \rightarrow \infty} \partial \ln \mathrm{E}_{t}\left[\left(1+X_{T}\right)^{a}\right] / \partial B_{t}=0$ if $\lim _{T \rightarrow \infty} \mathrm{E}_{t}\left[X_{T}^{a}\right]=$ 0 and $\lim _{T \rightarrow \infty} \partial \ln \mathrm{E}_{t}\left[\left(1+X_{T}\right)^{a}\right] / \partial B_{t}=a v$ if $\lim _{T \rightarrow \infty} \mathrm{E}_{t}\left[X_{T}^{a}\right]=\infty$.

First, changing the order of differentiation and expectation operators (see Billingsley 1995, Th. 16.8),

$$
\frac{\partial \ln \mathrm{E}_{t}\left[\left(1+X_{T}\right)^{a}\right]}{\partial B_{t}}=a v \frac{\mathrm{E}_{t}\left[X_{T}\left(1+X_{T}\right)^{a-1}\right]}{\mathrm{E}_{t}\left[\left(1+X_{T}\right)^{a}\right]}=a v\left(1-\frac{\mathrm{E}_{t}\left[\left(1+X_{T}\right)^{a-1}\right]}{\mathrm{E}_{t}\left[\left(1+X_{T}\right)^{a}\right]}\right) .
$$

Furthermore, according to part (i),

$$
\frac{\mathrm{E}_{t}\left[\left(1+X_{T}\right)^{a-1}\right]}{\mathrm{E}_{t}\left[\left(1+X_{T}\right)^{a}\right]} \sim \frac{\mathrm{E}_{t}\left[\left(1+X_{T}\right)^{a-1}\right]}{1+\mathrm{E}_{\mathrm{t}}\left[X_{T}^{a}\right]}
$$

Assume $a \geq 1$. As we have shown in case 1 of the proof of part (i), $X_{T}^{a-1} \leq\left(1+X_{T}\right)^{a-1} \leq$ $X_{T}^{a-1}+2^{a-1}+a-1$. If $\mathrm{E}_{\mathrm{t}}\left[X_{T}^{a}\right] \rightarrow \infty$, according to $(38), \mathrm{E}_{\mathrm{t}}\left[X_{T}^{a-1}\right] / \mathrm{E}_{\mathrm{t}}\left[X_{T}^{a}\right] \rightarrow 0$, which yields

$$
\lim _{T \rightarrow \infty} \frac{\partial \ln \mathrm{E}_{t}\left[\left(1+X_{T}\right)^{a}\right]}{\partial B_{t}}=a v
$$

Similarly, if $\mathrm{E}_{\mathrm{t}}\left[X_{T}^{a}\right] \rightarrow 0$, then, according to $(39), \lim _{T \rightarrow \infty} \mathrm{E}_{t}\left[X_{T}^{a-1}\right]=0$, which, according to part (i), implies that $\lim _{T \rightarrow \infty} \mathrm{E}_{t}\left[\left(1+X_{T}\right)^{a-1}\right]=1$ and

$$
\lim _{T \rightarrow \infty} \frac{\partial \ln E_{t}\left[\left(1+X_{T}\right)^{a}\right]}{\partial B_{t}}=0 .
$$

Next, consider the case of $0<a<1$. If $\mathrm{E}_{\mathrm{t}}\left[X_{T}^{a}\right] \rightarrow \infty$, because $\mathrm{E}_{\mathrm{t}}\left[\left(1+X_{T}\right)^{a-1}\right] \leq 1,(40)$ implies $\lim _{T \rightarrow \infty} \partial \ln \mathrm{E}_{t}\left[\left(1+X_{T}\right)^{a}\right] / \partial B_{t}=a v$.

Suppose that $\lim _{T \rightarrow \infty} \mathrm{E}_{\mathrm{t}}\left[X_{T}^{a}\right]=0$. By Markov's inequality, $\mathrm{P}_{t}\left[X_{T}>\epsilon\right] \leq \mathrm{E}_{t}\left[X_{T}^{a}\right] / \epsilon^{a} \rightarrow$ 0 , for any $\epsilon>0$. Similarly, $P_{t}\left[X_{T}<\epsilon\right] \leq \mathrm{E}_{t}\left[\left(1+X_{T}\right)^{a-1}\right] /(1+\epsilon)^{a-1}$. Thus, $1 \geq \mathrm{E}_{t}\left[\left(1+X_{T}\right)^{a-1}\right] \geq$ $P_{t}\left[X_{T}<\epsilon\right](1+\epsilon)^{a-1}$, and therefore $\liminf _{T \rightarrow \infty} \mathrm{E}_{t}\left[\left(1+X_{T}\right)^{a-1}\right] \geq(1+\epsilon)^{a-1}$ for any $\epsilon>0$. This implies that $\lim _{T \rightarrow \infty} \mathrm{E}_{t}\left[\left(1+X_{T}\right)^{a-1}\right]=1$, and therefore $\lim _{T \rightarrow \infty} \partial \ln \mathrm{E}_{t}\left[\left(1+X_{T}\right)^{a}\right] / \partial B_{t}=$ 0 .

We establish the long-run behavior of the quantities $S_{t}$ and $W_{r, t} / W_{n, t}$ for the case when $\gamma>1$ and $0<\eta<\eta^{\star}=2(\gamma-1)$. The results for all other regions in the parameter space 
can be obtained similarly.

The equilibrium stock price and the ratio of the individual wealth processes are given by

$$
\begin{aligned}
& S_{t}=\frac{\mathrm{E}_{t}\left[D_{T}^{1-\gamma}\left(1+\left(b \xi_{T}\right)^{\frac{1}{\gamma}}\right)^{\gamma}\right]}{\mathrm{E}_{t}\left[D_{T}^{-\gamma}\left(1+\left(b \xi_{T}\right)^{\frac{1}{\gamma}}\right)^{\gamma}\right]} . \\
& \frac{W_{r, t}}{W_{n, t}}=\frac{\mathrm{E}_{t}\left[D_{T}^{1-\gamma}\left(1+\left(b \xi_{T}\right)^{\frac{1}{\gamma}}\right)^{\gamma-1}\right]}{\mathrm{E}_{t}\left[D_{T}^{1-\gamma}\left(b \xi_{T}\right)^{\frac{1}{\gamma}}\left(1+\left(b \xi_{T}\right)^{\frac{1}{\gamma}}\right)^{\gamma-1}\right]} .
\end{aligned}
$$

We therefore need to characterize the long-run behavior of the following four quantities:

$$
\begin{aligned}
& \mathrm{E}_{t}\left[D_{T}^{1-\gamma}\left(1+\left(b \xi_{T}\right)^{\frac{1}{\gamma}}\right)^{\gamma}\right] \\
& \mathrm{E}_{t}\left[D_{T}^{-\gamma}\left(1+\left(b \xi_{T}\right)^{\frac{1}{\gamma}}\right)^{\gamma}\right] \\
& \mathrm{E}_{t}\left[D_{T}^{1-\gamma}\left(1+\left(b \xi_{T}\right)^{\frac{1}{\gamma}}\right)^{\gamma-1}\right] \\
& \mathrm{E}_{t}\left[D_{T}^{1-\gamma}\left(b \xi_{T}\right)^{\frac{1}{\gamma}}\left(1+\left(b \xi_{T}\right)^{\frac{1}{\gamma}}\right)^{\gamma-1}\right] .
\end{aligned}
$$

We will establish the asymptotic behavior of the stock price process in detail, the corresponding results for the wealth ratio process in Proposition 8 are obtained in a very similar fashion.

Consider the first expression,

$$
\mathrm{E}_{t}\left[D_{T}^{1-\gamma}\left(1+\left(b \xi_{T}\right)^{\frac{1}{\gamma}}\right)^{\gamma}\right]=D_{t}^{1-\gamma} \mathrm{E}_{t}\left[\left(\frac{D_{T}}{D_{t}}\right)^{1-\gamma}\left(1+\left(b \xi_{t} \frac{\xi_{T}}{\xi_{t}}\right)^{\frac{1}{\gamma}}\right)^{\gamma}\right]
$$

Given the aggregate dividend process,

$$
\left(\frac{D_{T}}{D_{t}}\right)^{1-\gamma}=e^{(T-t)\left(\mu(1-\gamma)-\frac{1}{2} \sigma^{2}(1-\gamma) \gamma\right)} e^{-\frac{1}{2}(1-\gamma)^{2} \sigma^{2}(T-t)+(1-\gamma) \sigma\left(B_{T}-B_{t}\right)} .
$$

As in the proof of Proposition 1, we introduce a new measure $Q$ with the Radon-Nikodym derivative

$$
\left(\frac{d Q}{d P}\right)_{t}=e^{-\frac{1}{2}(1-\gamma)^{2} \sigma^{2}(T-t)+(1-\gamma) \sigma\left(B_{T}-B_{t}\right)}
$$


By Girsanov's theorem, $B_{T}-B_{t}=B_{T}^{Q}-B_{t}^{Q}-(1-\gamma) \sigma(T-t)$, where $B_{t}^{Q}$ is a Brownian motion under the measure $Q$. Using the expression for $b$ from Proposition $1, b=e^{T(\gamma-1) \sigma^{2} \eta}$, we find

$$
\begin{aligned}
\mathrm{E}_{t}\left[D_{T}^{1-\gamma}\left(1+\left(b \xi_{T}\right)^{\frac{1}{\gamma}}\right)^{\gamma}\right]= & e^{T\left(\mu(1-\gamma)-\frac{1}{2} \sigma^{2}(1-\gamma) \gamma\right)+t\left(-\frac{1}{2} \sigma^{2}(1-\gamma)^{2}\right)+B_{t}(\sigma(1-\gamma))} \\
& \times \mathrm{E}_{t}^{Q}\left[\left(1+e^{\left(-\frac{1}{2} \eta^{2} \sigma^{2} \frac{1}{\gamma}\right) T+\left(\frac{1}{\gamma}(\gamma-1) \sigma^{2} \eta\right) t+\frac{\eta \sigma}{\gamma} B_{T}^{Q}}\right)^{\gamma}\right] .
\end{aligned}
$$

We will omit the superscript $Q$ in (41), since the distribution of $B_{t}^{Q}$ under the measure $Q$ is the same as the distribution of $B_{t}$ under the original measure $P$.

Using the assumption that $t=\lambda T$, define

$$
X_{T}=e^{\left(-\frac{1}{2} \eta^{2} \sigma^{2} \frac{1}{\gamma}+(1-\lambda) \frac{1}{\gamma}(\gamma-1) \sigma^{2} \eta\right) T+\frac{\eta \sigma}{\gamma} B_{T}} .
$$

We now apply the result of lemma 1, with

$$
\begin{aligned}
c & =-\frac{1}{2} \eta^{2} \sigma^{2} \frac{1}{\gamma}+(1-\lambda) \frac{1}{\gamma}(\gamma-1) \sigma^{2} \eta \\
v & =\frac{\eta \sigma}{\gamma} \\
a & =\gamma .
\end{aligned}
$$

Since we are assuming $\gamma>1$ and $0<\eta<2(\gamma-1), \lim _{T \rightarrow \infty} \mathrm{E}_{t}\left[X_{T}^{a}\right]=\infty$.

According to lemma 1,

$$
\mathrm{E}_{t}\left[D_{T}^{1-\gamma}\left(1+\left(b \xi_{T}\right)^{\frac{1}{\gamma}}\right)^{\gamma}\right] \sim e^{\left(\mu(1-\gamma)-\frac{1}{2} \sigma^{2}(1-\gamma) \gamma\right) T+\left(-\frac{1}{2} \sigma^{2}(\eta+1-\gamma)^{2}\right) t+\sigma(\eta+1-\gamma) B_{t}}
$$

We next examine

$$
\mathrm{E}_{t}\left[D_{T}^{-\gamma}\left(1+\left(b \xi_{T}\right)^{\frac{1}{\gamma}}\right)^{\gamma}\right]
$$

Using a similar change of measure, we find

$$
\begin{aligned}
\mathrm{E}_{t}\left[D_{T}^{-\gamma}\left(1+\left(b \xi_{T}\right)^{\frac{1}{\gamma}}\right)^{\gamma}\right]= & e^{\left(-\mu \gamma+\frac{1}{2} \sigma^{2}(1+\gamma) \gamma\right) T+\left(-\frac{1}{2} \sigma^{2} \gamma^{2}\right) t+(-\sigma \gamma) B_{t}} \\
& \times \mathrm{E}_{t}\left[\left(1+e^{\left(-\sigma^{2} \eta \frac{1}{\gamma}-\frac{1}{2} \eta^{2} \sigma^{2} \frac{1}{\gamma}\right) T+\sigma^{2} \eta t+\frac{\eta \sigma}{\gamma} B_{T}}\right)^{\gamma}\right] .
\end{aligned}
$$


We apply lemma 1 , setting $X_{T}=e^{c T+v B_{T}}$ and

$$
\begin{aligned}
c & =-\sigma^{2} \eta \frac{1}{\gamma}-\frac{1}{2} \eta^{2} \sigma^{2} \frac{1}{\gamma}+(1-\lambda) \sigma^{2} \eta \\
v & =\frac{\eta \sigma}{\gamma} \\
a & =\gamma
\end{aligned}
$$

The value of $\lim _{T \rightarrow \infty} \mathrm{E}_{t}\left[X_{T}^{a}\right]$ depends on the exact combination of the model parameters. In particular,

$$
\lim _{T \rightarrow \infty} \mathrm{E}_{t}\left[X_{T}^{a}\right]=\left\{\begin{array}{l}
\infty, \quad-2 \eta+\lambda\left(2 \gamma \eta-\eta^{2}\right)>0 \\
0, \quad-2 \eta+\lambda\left(2 \gamma \eta-\eta^{2}\right)<0
\end{array}\right.
$$

(see the proof of lemma 1, part (i)). Define

$$
\lambda_{S}=\frac{2}{2 \gamma-\eta}
$$

Note that, because $\gamma>1$ and $0<\eta<2(\gamma-1), 0<\lambda_{S}<1$. Then, $\lim _{T \rightarrow \infty} \mathrm{E}_{t}\left[X_{T}^{a}\right]=\infty$ if $\lambda>\lambda_{S}$ and $2 \gamma \eta-\eta^{2}>0$ or if $\lambda<\lambda_{S}$ and $2 \gamma \eta-\eta^{2}<0$, and the limit is equal to zero otherwise.

By lemma 1, if $\lim _{T \rightarrow \infty} \mathrm{E}_{t}\left[X_{T}^{a}\right]=\infty$,

$$
\mathrm{E}_{t}\left[D_{T}^{-\gamma}\left(1+\left(b \xi_{T}\right)^{\frac{1}{\gamma}}\right)^{\gamma}\right] \sim e^{T\left(-\mu \gamma+\frac{1}{2} \sigma^{2}(1+\gamma) \gamma-\sigma^{2} \eta\right) T+\left(-\frac{1}{2} \sigma^{2}(\eta-\gamma)^{2}\right) t+\sigma(\eta-\gamma) B_{t}}
$$

while if $\lim _{T \rightarrow \infty} \mathrm{E}_{t}\left[X_{T}^{a}\right]=0$, then

$$
\mathrm{E}_{t}\left[D_{T}^{-\gamma}\left(1+\left(b \xi_{T}\right)^{\frac{1}{\gamma}}\right)^{\gamma}\right] \sim e^{\left(-\mu \gamma+\frac{1}{2} \sigma^{2}(1+\gamma) \gamma\right) T+\left(-\frac{1}{2} \sigma^{2} \gamma^{2}\right) t+(-\sigma \gamma) B_{t}} .
$$

Using our definition of $\lambda_{S}$, we re-state these results as

$\mathrm{E}_{t}\left[D_{T}^{-\gamma}\left(1+\left(b \xi_{T}\right)^{\frac{1}{\gamma}}\right)^{\gamma}\right] \sim \begin{cases}e^{\left(-\mu \gamma+\frac{1}{2} \sigma^{2}(1+\gamma) \gamma\right) T+\left(-\frac{1}{2} \sigma^{2} \gamma^{2}\right) t-\sigma \gamma B_{t}}, & 0 \leq \lambda<\lambda_{S} \\ e^{\left(-\mu \gamma+\frac{1}{2} \sigma^{2}(1+\gamma) \gamma-\sigma^{2} \eta\right) T+\left(-\frac{1}{2} \sigma^{2}(\eta-\gamma)^{2}\right) t+\sigma(\eta-\gamma) B_{t}}, & \lambda_{S}<\lambda \leq 1\end{cases}$

Having established the behavior of both the numerator and the denominator of the expression for the stock price, we have proven the limiting result for the stock price itself. 
According to part (ii) of lemma 1, not only the stock price, but also the mean and volatility of returns behave according to the asymptotic expressions of Proposition 6 in the limit of the economy horizon $T$ approaching infinity. The same is true for the ratio of individual wealth and the volatility of that ratio in Proposition 8.

\section{Proof of Proposition 7}

When the financial markets are dynamically complete and there is a single source of uncertainty (driven by a Brownian motion), the fraction of the agent's wealth invested in stock can be computed as a ratio of the instantaneous volatility of the agent's wealth to the instantaneous volatility of the cumulative stock return process. Propositions 6 and 8 provide expression for the long-run behavior of the volatility of stock returns and individual wealth processes, from which the expression for portfolio holdings follow immediately. To decompose the portfolio holdings of the rational trader into as a sum of the myopic and hedging demands, we compute the hedging demand as $\mu_{S} /\left(\gamma \sigma_{S}^{2}\right)$, where $\mu_{S}$ and $\sigma_{S}$ are the drift and the diffusion coefficients of the stock return process. The difference between the total portfolio holdings and the myopic component define the agent's hedging demand. For the irrational trader, the calculations are analogous, except the myopic demand is given by $\hat{\mu}_{S} /\left(\gamma \sigma_{S}^{2}\right)=\left(\mu_{S}+\eta \sigma \sigma_{S}\right) /\left(\gamma \sigma_{S}^{2}\right)$, where $\hat{\mu}_{S}$ is the expected stock return as perceived by the irrational trader. 


\section{References}

[1] Alpert, M; Raiffa, H. 1959, A progress report on the training of probability assessors. Reprinted in D. Kahneman, P. Slovic, and A. Tversky (eds.) Judgement under Uncertainty: Heuristics and Biases. Cambridge: Cambridge University Press, 1982.

[2] Athey, Susan. 2002, Monotone Comparative Statics Under Uncertainty, Quarterly Journal of Economics, 117 (1), 187-223.

[3] Barberis, Nicholas; Shleifer, Andrei; Vishny, Robert. 1998, A Model of Investor Sentiment, Journal of Financial Economics, 49 (3), 307-43.

[4] Benartzi, Shlomo; Thaler, Richard H. 2001, Naive Diversification Strategies in Defined Contribution Saving Plans, American Economic Review, 91(1), 79-98.

[5] Billingsley, Patrick. 1995, Probability and Measure, Wiley-Interscience, Third Edition.

[6] Black, Fischer. 1986, Noise, Journal of Finance, 41 (3), 529-43.

[7] Black, Fischer; Scholes, Myron S. 1973, The Pricing of Options and Corporate Liabilities, Journal of Political Economy, 81 (3), 637-54.

[8] Blume, Lawrence; Easley, David. 2001, If You're So Smart, Why Aren't You Rich? Belief Selection in Complete and Incomplete Markets, Cowles Foundation Discussion Papers, 1319.

[9] Cecchetti, Stephen G.; Lam, Pok-Sang; Mark, Nelson C. 2000, Asset Pricing with Distorted Beliefs: Are Equity Returns Too Good to Be True?, American Economic Review, 90 (4), 787-805.

[10] Cox, John; Huang, Chi-fu. 1989, Optimal Consumption and Portfolio Policies when Asset Prices Follow a Diffusion Process, Journal of Economic Theory, 49, 33-83.

[11] De Long, J. Bradford; Shleifer, Andrei; Summers, Lawrence H.; Waldman, Robert J. 1990, Noise Trader Risk in Financial Markets, The Journal of Political Economy, 98 (4), 703-38.

[12] De Long, J. Bradford; Shleifer, Andrei; Summers, Lawrence H.; Waldman, Robert J. 1991, The Survival of Noise Traders in Financial Markets, Journal of Business, 64 (1), $1-19$. 
[13] Duffie, Darrell. 1996, Dynamic Asset Pricing Theory, Princeton University Press, Second Edition.

[14] Duffie, Darrell. 1986, Stochastic Equilibria: Existence, Spanning Number, and the 'No Expected Financial Gain from Trade' Hypothesis, Econometrica, 54 (5), 1116-84.

[15] Duffie, Darrell; Huang, Chi-fu. 1986, Implementing Arrow-Debreu Equilibria by Continuous Trading of Few Long-Lived Securities, Econometrica, 53 (6), 1337-56.

[16] Figlewski, Stephen. 1978, Market "Efficiency" in a Market with Heterogeneous Information, Journal of Political Economy, 86 (4), 581-97

[17] Friedman, Milton. 1953, The Case for Flexible Exchange Rates, Essays in Positive Economics, University of Chicago Press.

[18] Hakansson, Nils H. 1971, Capital Growth and the Mean-Variance Approach to Portfolio Selection, Journal of Financial and Quantitative Analysis, 6 (1), 517-557.

[19] Halmos, Paul R. 1950, Measure Theory, D. Van Nostrand Company, Inc., Tenth Printing.

[20] Harrison, J. Michael; Kreps, David M. 1979, Martingales and Arbitrage in Multiperiod Securities Markets, Journal of Economic Theory, 2 (3), 381-408.

[21] Hirshleifer, David; Subrahmanyam, Avanidhar; Titman, Sheridan. 2002, Feedback and the Success of Irrational Investors, Working Paper.

[22] Kahneman, Daniel; Tversky, Amos. 1979, Prospect Theory: An Analysis of Decision under Risk, Econometrica, 47(2), 263-91.

[23] Karatzas, Ioannis; Shreve, Steven E. 1991, Brownian Motion and Stochastic Calculus, Springer-Verlag New York, Inc., Second Edition.

[24] Loewenstein, Mark; Willard, Gregory A. 2002, Asset Pricing Puzzles and Market Clearing, Working Paper.

[25] Lucas, Robert E., 1978, Asset Prices in an Exchange Economy, Econometrica, 46 (6), $1429-45$.

[26] Odean, Terrance. 1998, Are Investors Reluctant to Realize Their Losses?, Journal of Finance, 53(5), 1775-98. 
[27] Radner, Roy. 1972, Existence of Equilibrium of Plans, Prices, and Price Expectations in a Sequence of Markets, Econometrica, 40 (2), 289-303.

[28] Sandroni, Alvaro. 2000, Do Markets Favor Agents Able to Make Accurate Predictions?, Econometrica, 68 (6), 1303-41.

[29] Shleifer, Andrei; Vishny, Robert W. 1997, The Limits of Arbitrage, Journal of Finance, $52(1), 35-55$.

[30] Shleifer, Andrei; Summers, Lawrence H. 1990, The Noise Trader Approach to Finance, Journal of Economic Perspectives, 4 (2), 19-33.

[31] Wang, Jiang. 1996, The Term Structure of Interest Rates in a Pure Exchange Economy with Heterogeneous Investors, Journal of Financial Economics, 41 (1), 75-110. 